San Jose State University

SJSU ScholarWorks

Master's Theses

Master's Theses and Graduate Research

1997

\title{
Gain prediction for multiple signals in erbium doped fiber amplifiers
}

Bruce Henderer

San Jose State University

Follow this and additional works at: https://scholarworks.sjsu.edu/etd_theses

\section{Recommended Citation}

Henderer, Bruce, "Gain prediction for multiple signals in erbium doped fiber amplifiers" (1997). Master's Theses. 1448.

DOI: https://doi.org/10.31979/etd.qjb9-chp3

https://scholarworks.sjsu.edu/etd_theses/1448

This Thesis is brought to you for free and open access by the Master's Theses and Graduate Research at SJSU ScholarWorks. It has been accepted for inclusion in Master's Theses by an authorized administrator of SJSU ScholarWorks. For more information, please contact scholarworks@sjsu.edu. 


\section{INFORMATION TO USERS}

This manuscript has been reproduced from the microfilm master. UMI films the text directly from the original or copy submitted. Thus, some thesis and dissertation copies are in typewriter face, while others may be from any type of computer printer.

The quality of this reproduction is dependent upon the quality of the copy submitted. Broken or indistinct print, colored or poor quality illustrations and photographs, print bleedthrough, substandard margins, and improper alignment can adversely affect reproduction.

In the unlikely event that the author did not send UMI a complete manuscript and there are missing pages, these will be noted. Also, if unauthorized copyright material had to be removed, a note will indicate the deletion.

Oversize materials (e.g., maps, drawings, charts) are reproduced by sectioning the original, beginning at the upper left-hand corner and continuing from left to right in equal sections with small overlaps. Each original is also photographed in one exposure and is included in reduced form at the back of the book.

Photographs included in the original manuscript have been reproduced xerographically in this copy. Higher quality 6" $\times 9$ " black and white photographic prints are available for any photographs or illustrations appearing in this copy for an additional charge. Contact UMI directly to order.

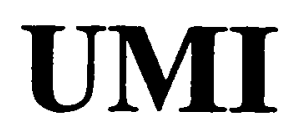

A Bell \& Howell Information Company 300 North Zeeb Road, Ann Arbor MI 48106-1346 USA

$313 / 761-4700 \quad 800 / 521-0600$ 



\title{
GAIN PREDICTION FOR MULTIPLE SIGNALS \\ IN ERBIUM DOPED FIBER AMPLIFIERS
}

\author{
A Thesis \\ Presented to \\ The Faculty of the Department of Electrical Engineering \\ San Jose State University
}

In Partial Fulfillment

of the Requirements for the Degree

Master of Science

by

Bruce Henderer

May 1997 
UMI Number: 1384697

UMI Microform 1384697

Copyright 1997, by UMI Company. All rights reserved.

This microform edition is protected against unauthorized copying under Title 17, United States Code.

\section{UMI}

300 North Zeeb Road

Ann Arbor, MI 48103 
APPROVED FOR THE DEPARTMENT OF ELECTRICAL ENGINEERING
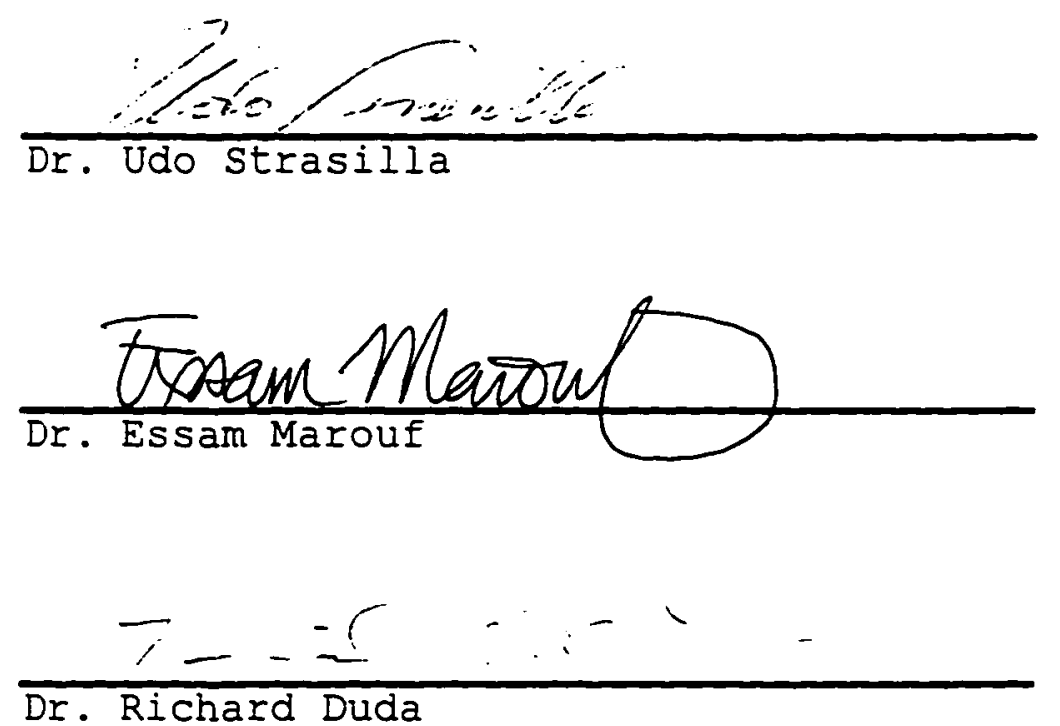

APPROVED FOR THE UNIVERSITY

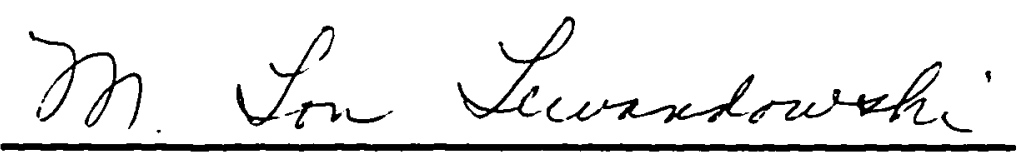


(C) 1997

Bruce Henderer

ALI RIGHTS RESERVED 


\section{ABSTRACT \\ GAIN PREDICTION FOR MULTIPLE SIGNALS \\ IN ERBIUM DOPED FIBER AMPLIFIERS \\ by Bruce Henderer}

A model for addressing the difficulty in predicting the gain in an Erbium Doped Fiber Amplifier (EDFA) in a Wavelength Division Multiplexed (WDM) optical communication system was developed and is presented in this thesis. The model takes into account gain tilt between channels present and keeps track of channel power and noise accumulation. An empirical test that develops the model's parameters is based on the fact that a specific gain is achievable for a channel irrelevant of the input power of all the channels present. The theory behind this phenomenon relates to absorption and emission cross sections as well as the extent of saturation of the doped fiber. Results show that the predictions of the model are within $0.5 \mathrm{~dB}$ of experimental values. 


\section{Acknowledgments}

I would like to thank the Lawrence Livermore National Laboratory for allowing me the opportunity to conduct my research at their facilities. I would like to extend my appreciation to all the people who have helped me in my endeavor.

I would like to thank my wife, Christy, for her enduring patience and her support. 


\section{Table of Contents}

Abstract

Acknowledgments

List of Figures

vii

List of Tables

viii

Chapter 1 Introduction

Chapter 2 Erbium Doped Fiber Amplifiers

Chapter 3 System Requirements

Chapter 4 Wavelength Division Multiplexing Testing and Results

Chapter 5 Explanation of the Convergence and Divergence Points

Chapter 6 Conclusion

Notes

Appendix A List of Acronyms

Appendix B Matlab Program for Model

Appendix C Table of Convergence Point Values

Appendix D LabVIEW Data Collection Program

Appendix E LabVIEW Model of EDFA 


\section{List of Figures}

Figure 1: Amplifier vs. Regenerators 2

Figure 2: Block Diagram of an EDFA 8

Figure 3: Energy Diagram of EDFA 10

Figure 4: Regions of an EDFA 13

Figure 5: Gain Tilt 15

Figure 6: WDM Experimental setup 23

Figure 7: Gain Curves for Two Wavelengths 25

Figure 8: Convergence of Gain Curves 26

Figure 9: Gain Curves for Three wavelengths 27

Figure 10: Two Signals vs. Three Signals 28

Figure 11: Experimental Link Setup 30

Figure 12: Four Cascaded EDFAs 32

Figure 13: Second Data Set for Cascade 32

Figure 14: Absorption and Emission Cross Section 38

Figure 15: Divergence of Gain 41 
List of Tables

Table 1: Output Power of Cascaded EDFAs

Table 1: Second Run, Output Power of Cascaded EDFAs 


\section{Chapter 1}

Introduction

Erbium Doped Fiber Amplifiers (EDFAs) are used in today's optical point-to-point and network links. Accurate modeling of these amplifiers enables optimum placement of the amplifiers within the link. A model is presented herein that accomplishes this task; only a single empirical test is required to provide the necessary parameters for the model. The need for a simple test for determining model parameters arises from the many vendors creating EDFAs.

Fiber-optic communication has been on the rise since the mid 1970's when optical fiber became sufficiently low loss and inexpensive to manufacture. Its enormous bandwidth compared to copper made it the choice of most telecommunications companies. To extend the range of optical signals, repeaters were constructed to conduct optical to electrical conversion, signal amplification, and retransmission as an optical data stream. Because of the speed limitations of electronic equipment, single wavelength communications still do not entirely utilize the enormous bandwidth of fiberoptic lines.

Using the principle that light of different frequencies can pass through the same medium without interfering has brought about wavelength division multiplexing (WDM). Multiple wavelengths of light are propagated through the 
same fiber at the same time. This made repeaters difficult to use since each wavelength required its own repeater. In addition, extra losses were incurred in the process of separating the wavelengths to be received by their individual repeaters.

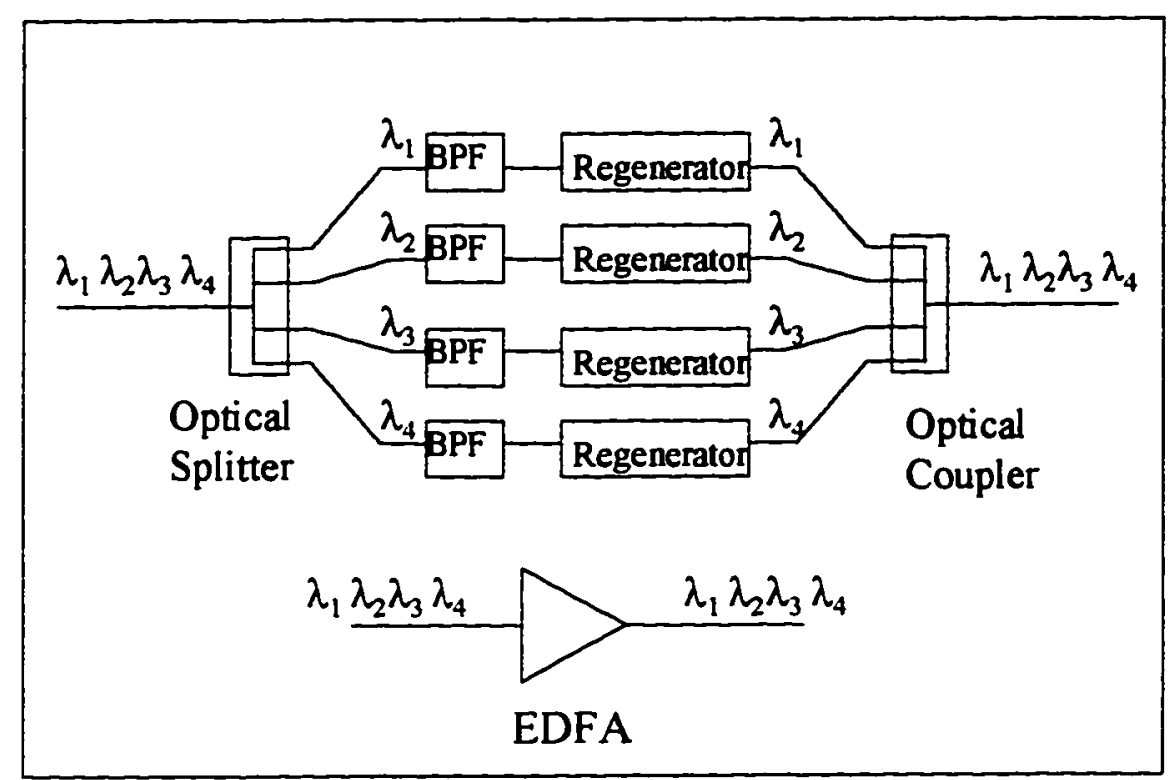

Figure 1: Amplifier Vs Regenerators

The EDFA conveniently solves these problems. It possess a wide optical bandwidth $(30+\mathrm{nm})$ and the capability of amplifying all signals in the passband simultaneously. Unlike electrical amplifiers and most semiconductor optical amplifiers, the EDFA's design makes multiple signal amplification virtually crosstalk free. The ability to amplify multiple signals reduces the loss incurred by the 
optical splitters and Band Pass Filters (BPF) required by repeaters. This increases the distance between amplifiers adding to cost savings. Additionally, the EDFA does not require the clock recovery circuit that the repeater needs to reshape the pulse. This make the EDFA popular during upgrades since it reacts to total optical power and not data rate. Popularity for the EDFA increased as the price for laser diodes used for pumping the EDFA has fallen.

With all the advantages come several modeling difficulties. The most significant difficulty is the gain that each signal wavelength receives as it transits the amplifier. Modeling for single wavelengths is fairly simple, but a phenomenon referred to as gain tilt occurs in the multiple wavelength amplification process. Gain tilt is the unequal amplification that occurs when more than one wavelength is present at the input of an EDFA.

Trying to predict the numerous variables introduced by EDFAs in a WDM environment places a challenge on any attempt to model the amplifier's behavior. No less challenging are the equations used by amplifier design engineers to determine the behavior of EDFAs. These equations exactly predict the noise and signal gain for the single wavelength situation. The following rate equation is well known to 
accurately predict results if the EDFA is considered a two level system. ${ }^{1}$

$\frac{d p_{k}^{=}}{d k}=\beta_{0} \sigma_{a} \int_{s} 2 \pi \frac{\rho_{r}}{\rho_{0}} \psi_{s i}(r)\left\{\frac{\left.\eta_{k}\left[\sum_{j} \frac{p_{j}^{-}+p_{j}^{-}}{1+\eta_{j}} \psi_{s j}(r)\right] \cdot\left[p_{k}+2 p_{o k}\right]-\left[1+\sum_{i} \frac{\eta_{j}\left(p_{j}^{-}+p_{j}^{-}\right)}{1+\eta_{j}} \psi_{s k}(r)\right] p_{k}\right]}{1+\sum_{j}\left(p_{j}^{-}+p_{j}^{-}\right) \psi_{s j}(r)}\right\} d r$

Parameters required for this calculation include: $\rho_{0}$ the peak dopant density, $\sigma_{a}$ the total absorption cross section at the wavelength of interest, $\rho_{r}$ the dopant density distribution, $\eta_{k}$ the ratio of absorption to emission crosssection at the wavelength of interest, and $p_{j}$ the signal power normalized to the saturation power. ${ }^{2}$ Obtaining these parameters from the vendor of an amplifier is very difficult since the information tends to be proprietary. In addition, the equation does not deal with multiple wavelengths.

The difficulty in obtaining exact parameters and determining new theoretical equations is unlikely, especially not having any reliable measurement results to compare the equations with. For any real time system, the computation time would be considerable, especially for multiple wavelengths and multiple amplifiers. Thus a model is devised to eliminate the need for extensive a priori 
knowledge of the EDFA parameters or new theoretical equations.

The model has a fast execution speed since it does not have to numerically solve complex equations. This allows for near real-time results to input changes. Real-time solutions allow the model to be used in a reconfigurable network to determine if the signal quality will be reduced below an acceptable level if a planned change occurs.

Empirical testing of the EDFA to determine the model parameters allows the model to be adapted to any vendor's amplifiers. A single empirical test is used to determine the model's parameters. These parameters will already include signal wavelength and signal power dependencies. Therefore, the signals present and their respective initial powers will be the only necessary input data for the model. Accurate predictions by the model result from the discovery of a gain convergence point for each wavelength present at the input to an EDFA. Gain convergence is that a wavelength will have a specific gain for a given amount of total input power to the EDFA. This gain is irrelevant of the contribution of the wavelength to the total input power. The final results show that the model is capable of predicting signal gain to within $\pm 0.5 \mathrm{~dB}$. A explanation for the existence of the convergence point is given at the 
end of the thesis. The convergence point is found to be a result of the relation between the absorption cross section and the emission cross section of the doped fiber. 


\section{Chapter 2}

\section{Erbium Doped Fiber Amplifiers}

Erbium Doped Fiber Amplifiers (EDFAs) operate under the same principles that lasers do. The major differences between the two are a result of the purpose of each device. Lasers are designed to emit one wavelength and subdue all others while the EDEA's design amplifies a broad spectrum of wavelengths. Both amplify the number of photons at a given wavelength through the process of stimulated emission. The laser uses a cavity to ensure that a single wavelength is amplified but the EDFA has no reflective surfaces to produce this cavity. The EDFA produces all of its gain in a single pass. Therefore, the noise associated with the optical amplification process, which would be minimal in a laser due to the need for multiple passes through the cavity, is also larger in EDFAs.

EDFAs are constructed of three parts: a pump laser, an optical coupler, and a length of single mode fiber doped with the rare earth element Erbium. The optical coupler has two fiber inputs that are fused together into one fiber. The pump laser is a laser diode of wavelength $980 \mathrm{~nm}$ or 1480 nm. These two wavelengths are most useful as they correspond to the two most efficient absorption ranges by the Erbium ion. The $1480 \mathrm{~nm}$ wavelength advantage over the 
$980 \mathrm{~nm}$ wavelength pump source is based in the relative sensitivity of the EDFA to the wavelength of the pump laser. The EDFA is less sensitive to variations in the wavelength in the $1480 \mathrm{~nm}$ range providing for less strict tolerances in the pump laser requirements. ${ }^{3}$ The light weight and inexpensive nature of semiconductor laser diodes makes them popular as pump sources.

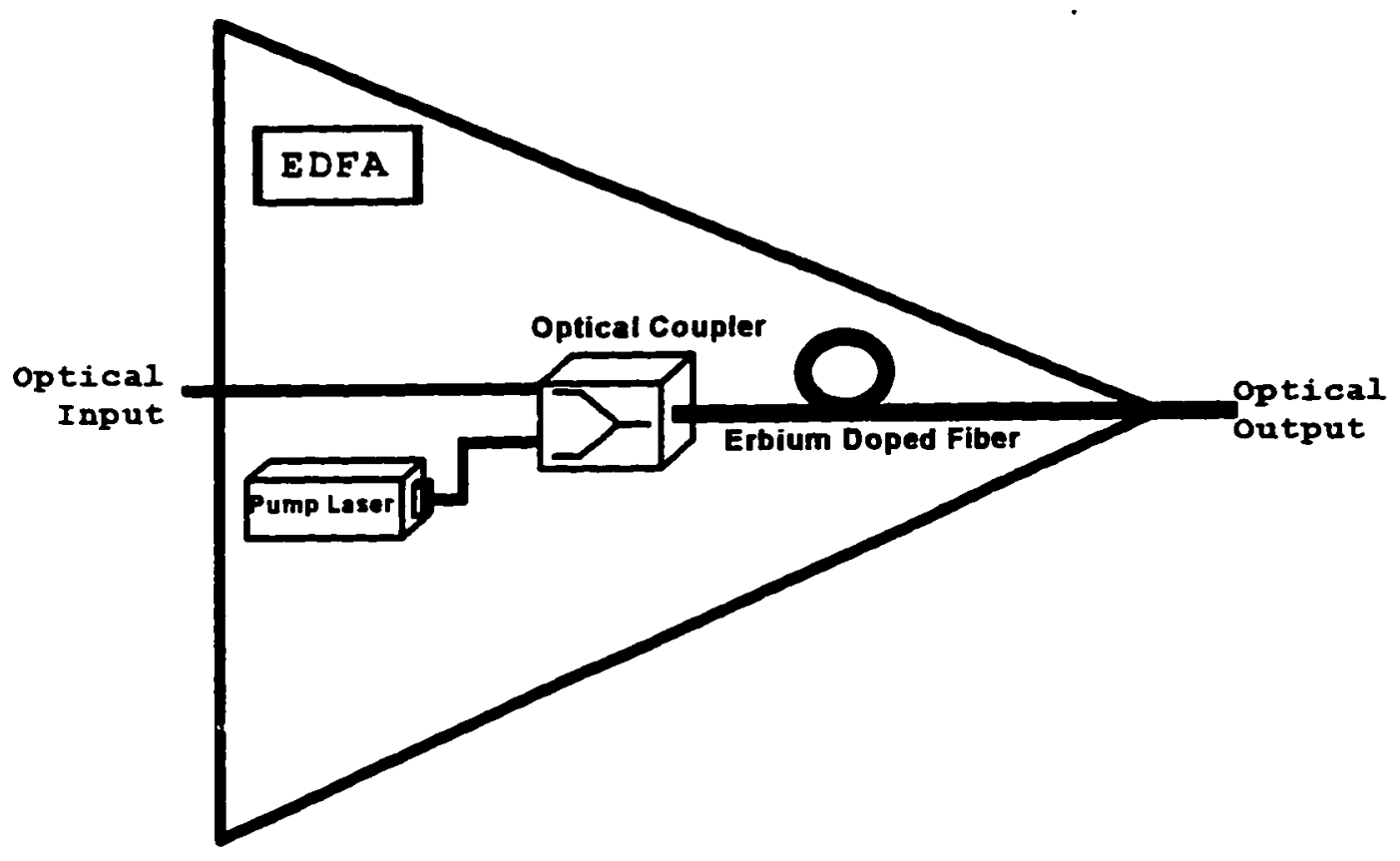

Figure 2: Block Diagram of an EDFA

The doped fiber amplifies wavelengths near the $1550 \mathrm{~nm}$ region of the optical spectrum based on the emission spectrum of $\mathrm{Er}^{3+}$. The Erbium ion can also efficiently absorb photons at these wavelengths. It is possible for a laser with a wavelength near the amplification band to be used to pump the medium into inversion. This laser wavelength would 
pump the EDFA but would be amplified like a signal wavelength. This creates an inefficient system as power given to the pump is emitted as an unwanted signal instead of being completely absorbed. This tendency becomes important in considering a WDM system as there are several wavelengths present. The characteristics of the EDFA are not equal for each wavelength indicating that pumping occurs by signal wavelengths.

The amplifying medium is nothing more than a piece of normal optical fiber that is doped with the rare earth element Erbium (Er) during fabrication. Within the glass substrate the Erbium ions are found nested between the atoms of the SiO= glass atoms." Sodium or Aluminum ions are doped into the glass to loosen the glass structure, facilitating the incorporation of the large Erbium ion. The active electrons in the Erbium ion that are used for amplification are not the outer valence electrons but rather an internal shell. The electronic configuration of Erbium is [Xe]4fN$1 / 5 s 2 / 5 p 6 / 6 s 0$. The amplifying transition is $4 f-4 f$. The outer $5 s$ and $5 p$ shells shield the inner $4 f$ shell from external fields. Thus the $5 \mathrm{~s}$ and $5 \mathrm{p}$ shells interact with the glass but the $4 \mathrm{f}$ shells are isolated from the glass providing a stable amplifying transition. 
The EDFA, on a quantum level, is sufficiently described by a three level system. The active electrons are elevated from the ground state by the pump laser. These electrons elevate two energy levels, from $N_{\text {: }}$ to $\mathrm{N}_{3}$, by the absorption of the energy provided by the pump laser (see Figure 3). The electrons decay to the $\mathrm{N}_{2}$ excited level in several microseconds without emitting optical energy. The energy is absorbed by the medium. The elevated electrons stay in the $\mathrm{N}$ : level for hundreds of microseconds to 1 millisecond.

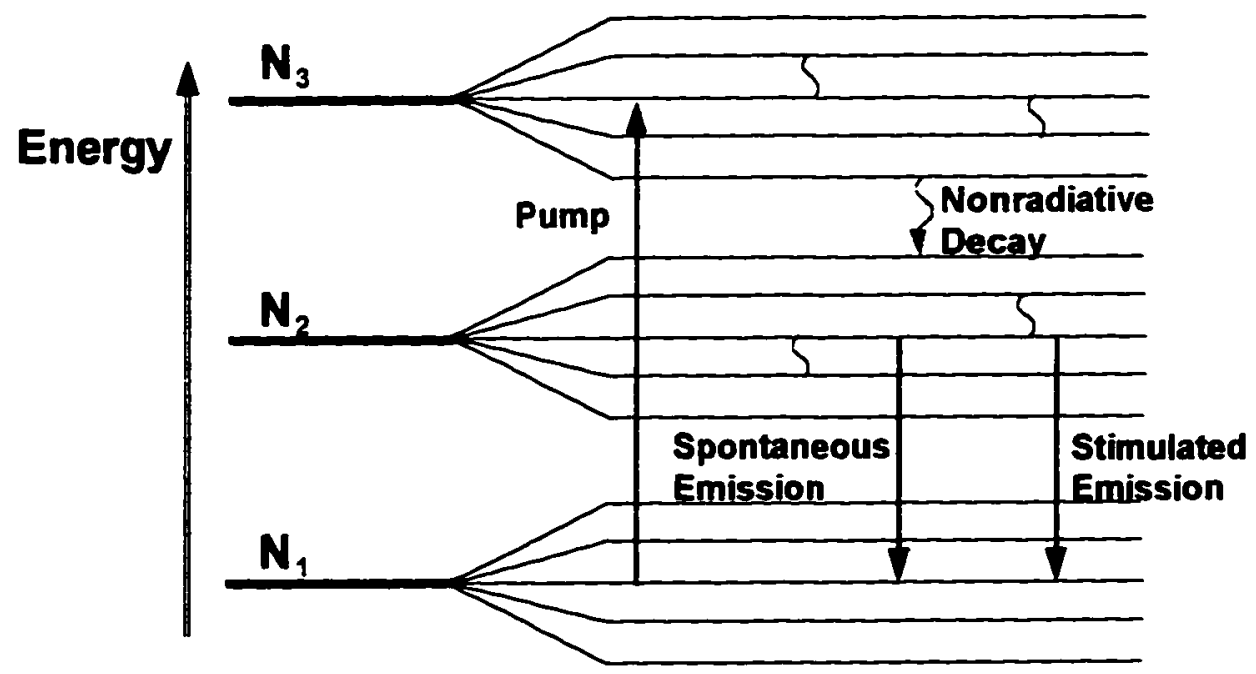

Eigure 3: Energy Diagram of EDFA

There are two methods for the final decay of the elevated electrons. A photon in the $1525 \mathrm{~nm}$ to $1575 \mathrm{~nm}$ region will interact with the electron causing it to decay, emitting a second photon. This photon will be in phase and have the exact wavelength as the original photon, both of 
which propagate in the medium through the same direction. This is the process of stimulated emission. As the two photons continue through the EDFA, they each interact with another electron stimulating two new photons. This avalanche process continues throughout the entire length of doped fiber.

A second means of decay for the excited electrons is spontaneous emission. An electron in an excited state will always try to return to its ground state. After a period of hundreds of microseconds the electron will decay from the $\mathrm{N}$ : state to the ground state emitting a photon. This photon will be of no particular phase with respect to any other photons present. The wavelength of a spontaneous photon is independent of any other photons present but is constrained to $1525 \mathrm{~nm}$ to $1575 \mathrm{~nm}$ by the band gap energy. This photon propagates and interacts with elevated electrons and stimulates emission. This is amplified spontaneous emission (ASE). It is the optical noise produced by an optical amplifier.

Ideally, an electron decaying from one energy level to another will emit a photon with exactly the amount of energy equal to the difference of the energy levels. Since the EDFA is an optically broadband device, another phenomenon must occur that causes the primary energy levels to vary. 
This is a result of stark splitting. Within the glass substrate are electric fields caused by its structure. These fields add or subtract energy from individual energy levels and result in the electrons having a multiple of possible energy levels, as shown Eigure $3 .^{\text {? }}$ The effect is termed homogeneous broadening.

The diagram suggests that there are a discrete number of transitions possible for the electron. The output spectrum of an EDFA is a continuous curve, not a handful of discrete Iines, suggesting that the above diagram is not exact in its representation. The additional effect is the result of inhomogeneous broadening. Inhomogeneous broadening is the variation of crystalline fields, random fields and lattice vibration that differ from one erbium site to another. ${ }^{8}$ The variations cause each stark split energy level to be further degenerated into near infinite energy levels.

other important considerations affecting the operation of an optical amplifier are optical power dependence and inter-signal crosstalk. The EDFA has two regions of operation which are specifically determined by the amount of input optical power. There is a saturated and an unsaturated region which are very similar to the same regions in an electrical amplifier. For small optical input 
signals the amplifier operates in the unsaturated region. This region is characterized by the highest gain capable by the EDFA and also the most optical noise production. When the maximum gain produced by the amplifier is reduced by 3 $\mathrm{dB}$, the amplifier is in the saturated region. This region is characterized by lower gain, reduced optical noise, and nearly constant signal output power.

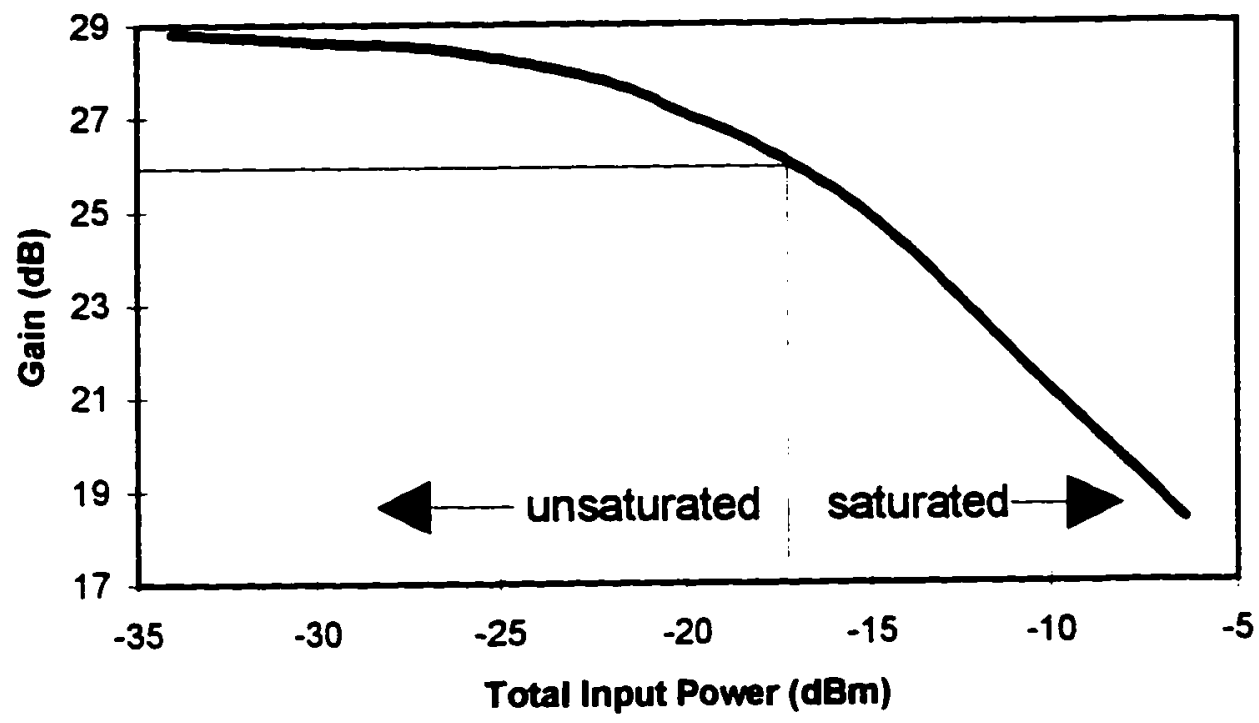

Figure 4: Regions of an EDFA

The cause of EDFA saturation is the limited number of excited electrons available for stimulated emission. For lower optical input powers, the number of electrons stimulated to emit photons is far smaller than the total number of elevated electrons. This leaves a large percentage of the excited electrons for spontaneous emission 
and to amplify the spontaneously emitted photons, i.e. large optical noise. Also, the large number of electrons compared to input photons insures that every input photon as well as stimulated photons are amplified resulting in large gain.

In the saturated region, the opposite is true. Most of the electrons are used for stimulated emission leaving few electrons for spontaneous emission. This reduces the noise output. The limit of the output power is controlled by the total number of elevated electrons within the doped fiber, most of which are used to amplify the signal. The limited amount of output power also implies a reduction in gain as input signal strength increases.

In the saturated region of the amplifier, the amount of total gain available is reduced. This is still the case with multiple wavelengths. The amplifier can only put out so much power. In the WDM environment, the output power is shared by all wavelengths present, limiting the gain of any individual wavelength.

The wavelengths present also have an effect on the individual wavelengths' gains. This is known as gain tilt. A readily observable phenomenon, gain tilt is the unequal gain of wavelengths that favors the longer wavelengths in the typical amplification band of the EDFA. The term 'tilt' 
comes from the slope observed by connecting the signals' optical power values.

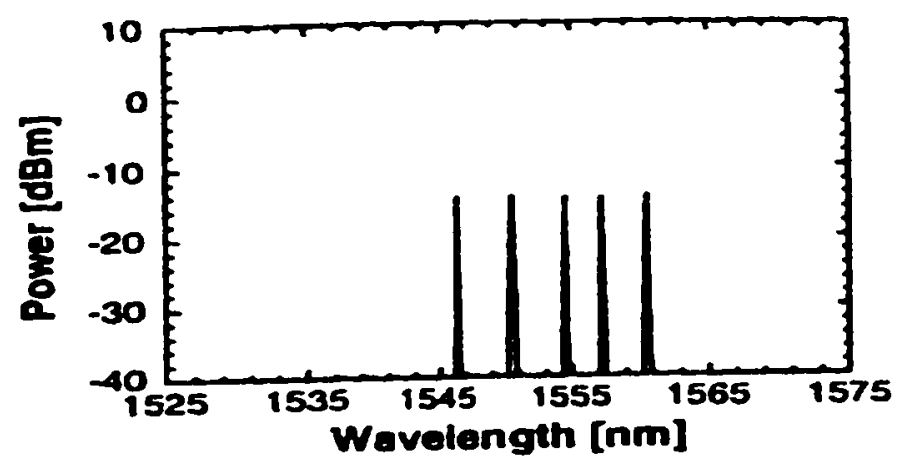

(a)

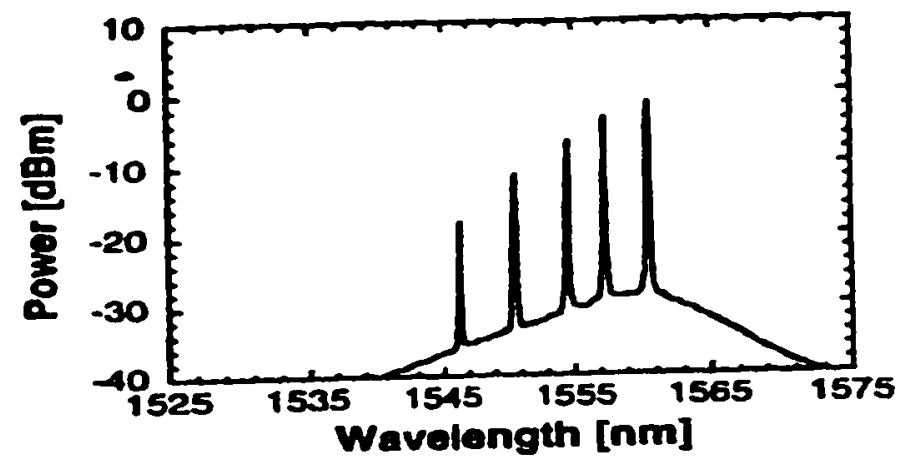

Figure 5: Gain Tilt

The issue of crosstalk is paramount to any discussion on amplifiers that need to amplify multiple signal wavelengths. Erbium doped fiber amplifiers are intrinsically immune to crosstalk. EDFA saturation and recovery times are on the order of $100 \mu \mathrm{s}$ to $1 \mathrm{~ms}$. When compared to the high data rates typical to optical communications, less than 10 ns bit periods, the EDFA appears to be unchanging. ${ }^{10}$ A single bit of a communication 
stream, whether a 'I' or a '0', will have almost no effect on the gain characteristics of the amplifier. Only the average power of the data stream effects the EDFA gain characteristics since it is the only parameter that has a period greater than the saturation/recovery time. 


\section{Chapter 3}

\section{System Requirements}

The communication link in which the EDFAs are to operate, designates the needed parameters that the model must keep track of. The system requirements are based on several equations. The equations usually quoted in books are based on the gain of the EDFA used in the system. This comes as a result of placing the EDFA directly before the detector in a system as a preamplifier with no other amplifiers in the link. The method is derived from two primary equations, one which determines Amplified Spontaneous Emission (ASE) noise and the other determines the Bit Error Rate (BER).

The following equation is used to predict the ASE noise production at the wavelength of the signal

$$
S=G n_{s p} h v
$$

where $n_{s F}$ is related to the noise figure of the amplifier and $v$ is the optical frequency of the signal:1. The $n_{s y}$ term is only weakly affected by gain and can be considered constant for this discussion. The gain of the amplifier is the only variable in the above equation.

The BER of a system is predictable by several equations. The primary equation for BER determination is 
related to the $Q$ factor by the following well known equation:

$$
B E R=\frac{\exp \left(\frac{Q^{2}}{2}\right)}{Q \sqrt{2 \pi}}
$$

Q for a system containing EDFAs is given by:

$$
Q=\frac{I^{2}}{\sigma_{t h}^{2}+\sigma_{s h}^{2}+\sigma_{s i-s p q}^{2}+\sigma_{s p-s p}^{2}}
$$

where

$$
\begin{gathered}
\sigma_{t h}^{2}=\frac{4 k T B e}{R_{L}} \\
\sigma_{s h}^{2}=4 R q B_{e} M F_{x}\left(G P_{t n}+P_{a s e}\right) \\
\sigma_{s g-s p}^{2}=4 \mathbb{R}^{2} B_{e} M G P_{t n} P_{a s e} / B_{o} \\
\sigma_{s p-s p}^{2}=4 R^{2} M P_{a s e}^{2} \frac{B_{e}}{B_{o}^{2}}\left(B_{o}-\frac{B_{e}}{2}\right)
\end{gathered}
$$

$B_{3}$ and $B_{0}$ are electrical and optical bandwidth respectively, both in $\mathrm{Hz}, \boldsymbol{R}$ is the responsivity of the receiver and ' $\mathrm{q}$ ' is the charge of an electron. $P_{\text {ir. }}$ is the optical signal power into the pre-amplifier and $P_{a s s}$ is the optical noise power. $G$ is the gain produced by the amplifier. $M$ is a multiplying factor related to the receiver gain. For PIN receivers its value is 1 . For Avalanche Photodiodes (APD) it is the value of the APD's gain. $F_{*}$ is related to $M$ and is: 


$$
F_{e x}=k\langle M\rangle+(1-k)\left(2-\frac{1}{\langle M\rangle}\right)
$$

where $k$ is the ionization ratio of the receiver and $\langle M\rangle$ is the average APD multiplier factor.

Equation $5 a$ is the familiar thermal (Johnson) noise term. The second term is shot noise developed in the receiver. The last two equations, equations $5 c$ and $5 d$, relate to a mixing phenomenon in the receiver. Energy at different wavelengths (frequencies) mixes together and generates fluctuations called "beating.": Equation $5 \mathrm{c}$ is the signal beating with the ASE noise and equation $5 d$ is the broad band ASE beating with itself.

In a communications link, determining the system gain can be cumbersome. Making the calculations for BER based on received power, instead of launch power and gain, is far more reasonable. Substituting equation 2 into equation 5 and converting input power times gain into received power results in the following modified noise equations:

$$
\begin{gathered}
\sigma_{t h}^{2}=\frac{4 k T B e}{R_{L}} \\
\sigma_{s h}^{2}=4 R q B_{e} M F_{x}\left(P_{s g}+P_{a s e}\right) \\
\sigma_{s g-s p}^{2}=4 R^{2} B_{e} M P_{s g} P_{a s e} / B_{o} \\
\sigma_{s p-s p}^{2}=4 R^{2} M P_{a s e}^{2} \frac{B_{e}}{B_{o}^{2}}\left(B_{o}-\frac{B_{e}}{2}\right)
\end{gathered}
$$


In these equations the only unknowns are the signal power and the noise power at the signal wavelength. The number of amplifiers and the losses between EDFAs need not be known for the calculation of the Bit Error Rate (BER). Therefore, signal and noise power are the primary pieces of information that need to be propagated through a link. With this data, the BER can be calculated and the link can be tested for different configurations. Any prediction method must produce these two pieces of information. Since the noise power at any wavelength can be determined from its gain and the final signal power is related to gain of the signal, gain tracking is of paramount importance to a cascaded EDFA link. 


\section{Chapter 4}

Wavelength Division Multiplexing Testing and Results

A study of the gain produced by an EDFA is important since it is the single necessary parameter for both EDFA noise production and signal power. These ultimately lead to the ability to estimate BER and in turn, Iink quality. The interactions between wavelengths in a WDM system need to be understood. How the gain varies due to the wavelengths present in the system and their corresponding optical powers is vital. Analysis of the data from testing provides valuable information that is useable for predictive modeling.

Selection of signal wavelengths is achieved by availability and usefulness. The useful optical bandwidth of the EDFA used is approximately $14 \mathrm{~nm}$, from $1546 \mathrm{~nm}$ to $1560 \mathrm{~nm}$. Within this region the gain tilt is apparent but manageable. This is sufficient bandwidth for four channels separated by $4 \mathrm{~nm}$ or eight channels at $2 \mathrm{~nm}$ separation. The test wavelengths used were separated by 4 nanometers, resulting in four channels in the bandpass of the EDFA. The separation of channels not only exaggerates the gain tilt between wavelengths, it also allows for covering the entire bandwidth of the amplifier. 
The following wavelengths are used for the testing: $1533 \mathrm{~nm}, 1546 \mathrm{~nm}, 1550 \mathrm{~nm}, 1554 \mathrm{~nm}$ and $1558 \mathrm{~nm}$. These wavelengths span the EDFA region of interest and include the ASE 'hump' at $1533 \mathrm{~nm}$. The data taken at the $1533 \mathrm{~nm}$ wavelength is necessary for determining the 'hump' size on the ASE spectrum in single wavelength, cascaded EDFA links. Figure 6 shows the setup for testing the EDFA in a WDM environment. The optical coupler combines the various signals that are present into a single fiber. The DiCon Dual Switch is computer controlled and has two settings. The first setting passes the incoming signal directly to the Optical Spectrum Analyzer (OSA). The second setting sends the incoming signals to the EDFA under test and takes the amplified output back to the OSA. The OSA is the HP 70951A Optical spectrum Analyzer and the variable attenuator is the JDS Fitel HA9 Optical Attenuator.

The controller computer is a Macintosh with a GPIB card installed. A LabVIEW program controls the timing of the optical switching by the Dual switch, the variable attenuator, and records the data collected by the OSA. The program removes the losses incurred by the DiCon Switch, resulting in the data being the true input and output of the EDFA. The program is then able to calculate total input power and gain for each wavelength present. 


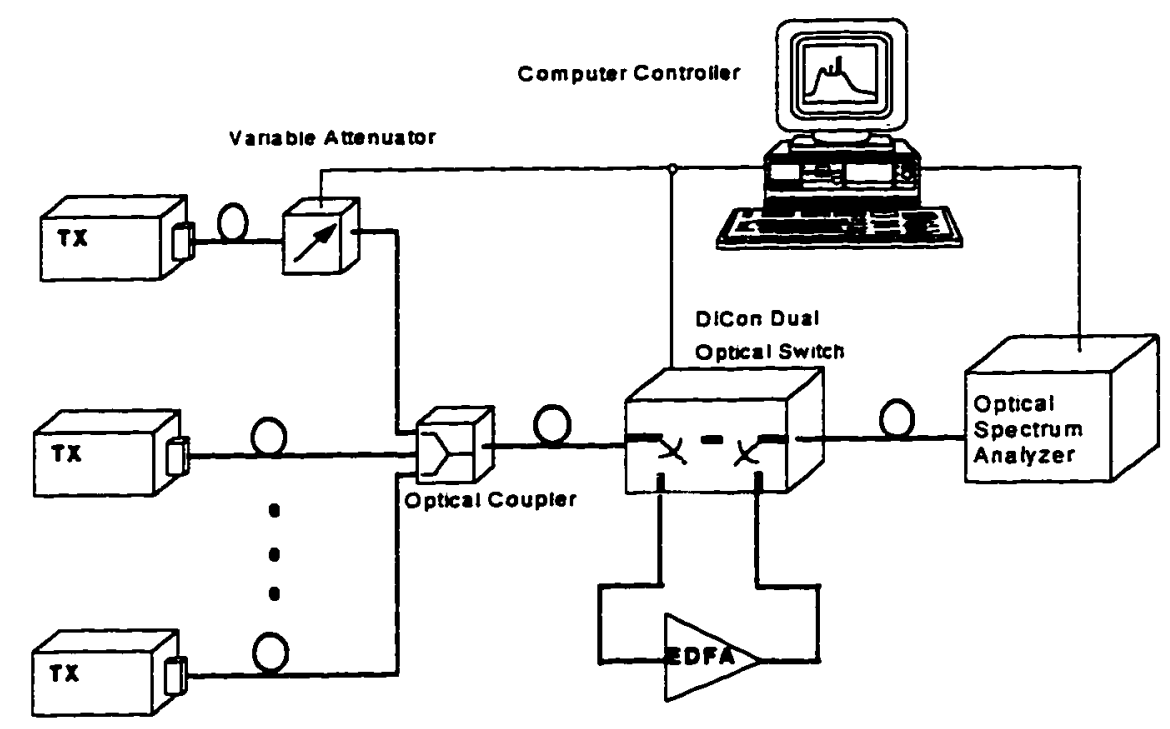

Figure 6: WDM Experimental Setup

The boxes labeled 'TX' in Figure 6 are the laser transmitters. They consist of an HP 8168A Tunable Laser set to $1550 \mathrm{~nm}$, three Nortel OC48 transmitters at $1533 \mathrm{~nm}, 1546$ $\mathrm{nm}$, and $1558 \mathrm{~nm}$. A DFB Laser Diode was tuned to $1554 \mathrm{~nm}$ for the remaining wavelength.

With the wavelengths selected, the only remaining variable is the amount of signal power present in each wavelength. The attenuator is used to control one wavelength's power. The power is attenuated until it has little effect on the system, usually less than $-30 \mathrm{dBm}$, to a power which dominated the amplifier, usually $10 \mathrm{~dB}$ higher than any other wavelength. The power variation is such that the signal goes from not affecting the gain of the other signals to dominating the response of the system. The very 
high power helps to stretch out the gain curve for later extrapolations.

The test method is to set the shorter wavelength(s) to a power that saturates the EDFA. The longest wavelength present is swept from low power to high power. The exact amount of the low power and the high power are not required but should extend well beyond the normal range of the operating system. The choice of sweeping the longer wavelength was arbitrary. A separate discussion and test system based on sweeping shorter wavelengths could have been used. In the end, the results will be the same. The steps taken by the model to predict the outcome must correspond to the choice of method.

Two wavelengths are tested in this way, $1558 \mathrm{~nm}$ and $1533 \mathrm{~nm}$. The unswept wavelength, $1533 \mathrm{~nm}$, was selected intentionally for the figure as it exaggerates the effect of signal power on signal gain and gain tilt. The total amplifier gain is included for reference and is defined as being the total output power of all signals present divided by the total input power of all signals present (in Watts). This line is constant no matter what combinations of wavelengths and powers are present.

The $1533 \mathrm{~nm}$ signal obtains less gain as the power of the $1558 \mathrm{~nm}$ signal increases. The gain tilt is obvious 
since the longer wavelength always has a larger gain than the shorter wavelength no matter what combination of input powers are used. The important part of Figure 7 is the extension of the $1533 \mathrm{~nm}$ signal's gain curves (see Figure 8). They appear to be converging. By taking a linear approximation of the curves and extending them, they converge to a point. I refer to this point as the power independent gain convergence point or convergence point. This point occurs for any power level of the shorter wavelength.

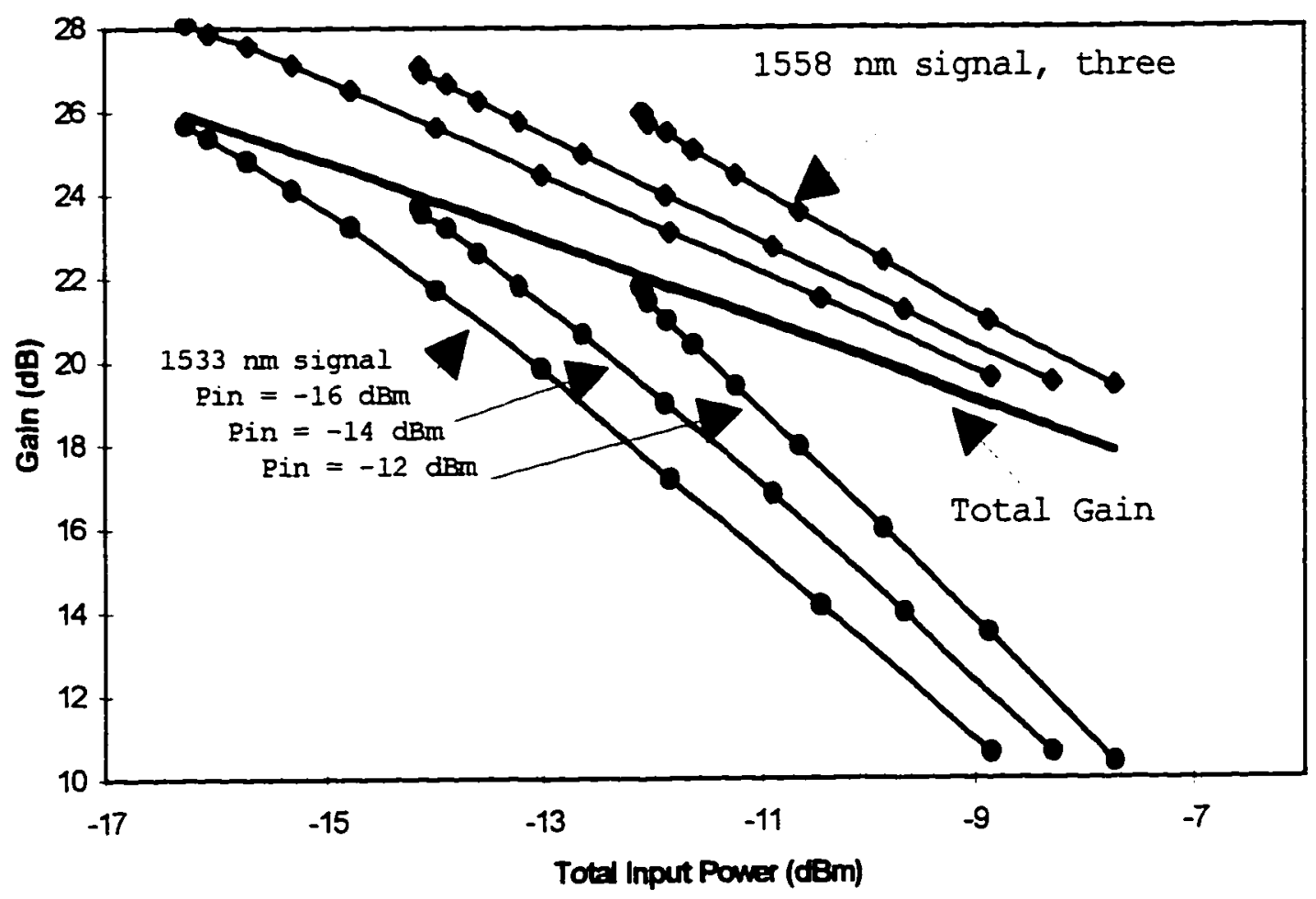

Figure 7: Gain Curves for Two Wavelengths 
To test this theory, several additional starting power levels of the $1533 \mathrm{~nm}$ signal were used and the $1558 \mathrm{~nm}$ signal power was swept from low power to high power. The gain curves of all the tests were extended through linear interpolation and the resulting graph of these interpolations clearly shows that all the curves intersect at a single point (see Figure 8 ).

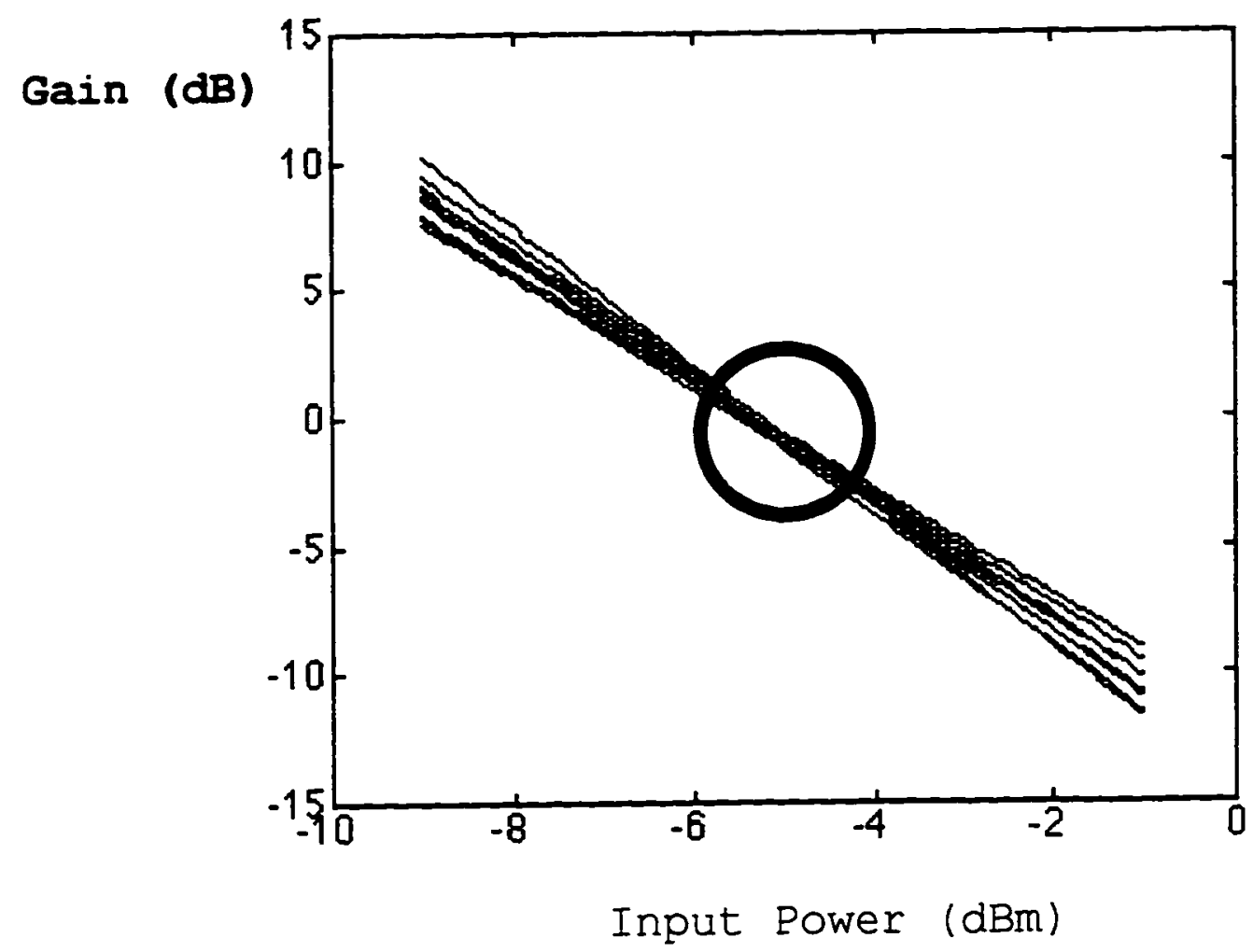

Figure 8: Convergence of Gain Curves

The other significant observation of

Figure 7 is the gain of the $1533 \mathrm{~nm}$ wavelength when the longer wavelength is minimized. Projecting the gain curve backwards, it appears to intersect the total gain curve at 
the value of the signal power of the shorter wavelength. This is reasonable. As the longer wavelength power is reduced, the system ultimately becomes a single wavelength EDFA link. The gain for a single wavelength through an EDFA in saturation is wavelength independent and is determined by the total gain curve.

The test procedure was repeated for the case of three wavelengths: $1533 \mathrm{~nm}, 1550 \mathrm{~nm}$, and $1558 \mathrm{~nm}$. The two shorter wavelengths' powers are set to a value and the longer

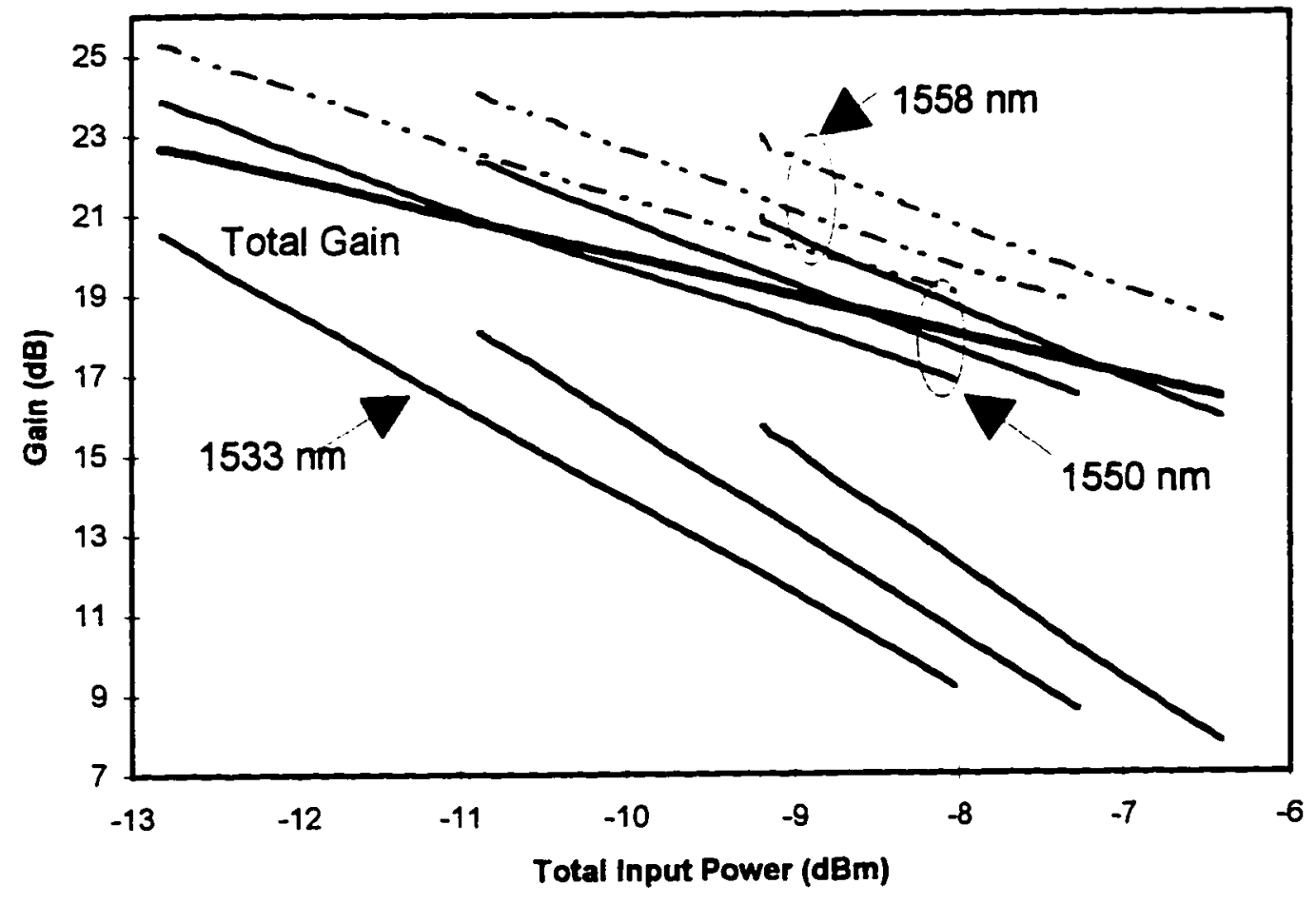

Figure 9: Gain Curves for Three Wavelengths wavelength $(1558 \mathrm{~nm})$ is swept, similar to the two wavelength 
experiment. Again the shorter wavelength's gain curves converge as seen in Figure 9. Each of the shorter wavelengths converge to separate points. Also, the shortest wavelength has a different convergence point for 3 wavelengths present than for 2 wavelengths present.

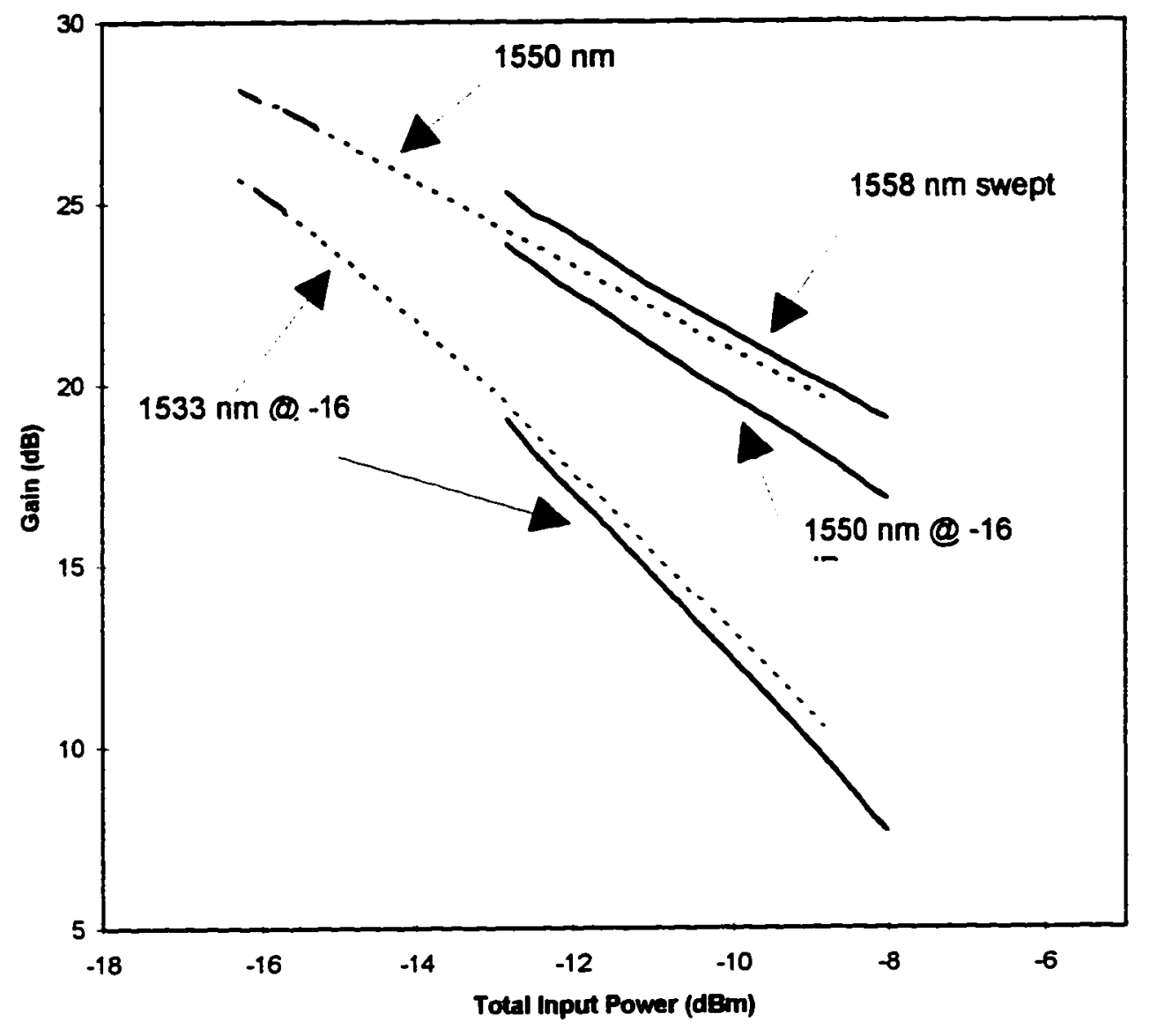

Figure 10: Two Signals vs. Three Signals

Figure 10 shows the results of a two wavelength test and a three wavelength test using the two wavelengths from the first test. The two wavelengths from the first test are held at a constant power in the second test. The divergence 
point and convergence point are scaleable to multiple wavel angths. The shortest wavelength is seen diverging from its gain curve developed in the first test. Likewise the second wavelength is seen diverging from the gain curve that resulted from the first test.

Modeling becomes a recursive process since the divergence point occurs for any combination of wavelengths and the convergence point can be calculated. Each combination of wavelengths to be used is assembled. The shorter wavelength(s) is(are) held constant and the longest wavelength is increased in power from a negligible power to a large power level. The shorter wavelength(s) are set to different power levels and the process repeated. Four tests are conducted to produce triangulation of the convergence point. The test is repeated for each wavelength in the useful band of the EDFA with the $1553 \mathrm{~nm}$ signal to provide data for single signal wavelength propagation.

The gain of each of the shorter wavelengths is extended through linear extrapolation to provide the convergence point. This point is then recorded for use in the model. Since the gain divergence is linear, it is a simple matter for the model to calculate the line between the divergence point and the convergence point. Convergence points are required for all wavelengths except the longest 
present. Each combination of wavelengths gets its own set of convergence points (see Appendix C for values).

The model starts with the two shortest wavelengths present and calculates the gain of the shorter wavelength. This is accomplished though the use of the convergence point calculated for the two wavelengths. If only two wavelengths are present, then the second wavelength receives the remainder of the power from the EDFA. A third wavelength will cause the gain of the shortest wavelength to diverge from the one calculated towards the convergence point determined for the second wavelength when the three wavelengths are present.

The process continues until all wavelengths are accounted for. The gain is then determined and the output power of the signal in question is known. This power is subtracted from the total output power of the EDFA. Now the

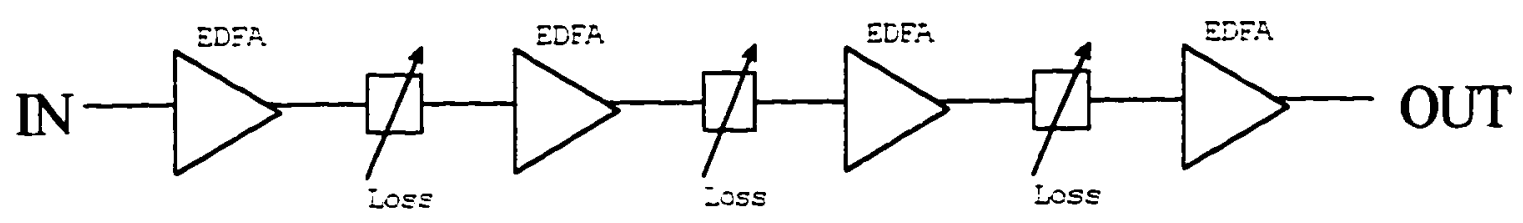

Figure 11: Experimental Link Setup process is repeated for a new total gain curve produced by the reduced output power. The short wavelength previously calculated is ignored and the convergence points used are those for the remaining wavelengths only. 
Using this method, the following gain curves are created comparing the model's prediction and experimental results for cascaded EDEAs. For the first experiment the losses between amplifiers was $20 \mathrm{~dB}$. The second experiment changed the losses between the amplifiers to $25 \mathrm{~dB}$. Figure 11 is the experimental setup for the tests. The OSA was used to measure values at the output of the individual EDFAs. The input value of the signals to the cascade was put into the model. The following are the experimental results and the model predictions. All tabulated values are in $\mathrm{dBm}$.

\begin{tabular}{|c|c|c|c|c|c|c|}
\hline Amplifier & 1550 Exp & $\begin{array}{c}1550 \\
\text { Model }\end{array}$ & 1554 Exp & $\begin{array}{c}1554 \\
\text { Model }\end{array}$ & 1558 Exp & $\begin{array}{c}1558 \\
\text { Model }\end{array}$ \\
\hline 1 & 4.2 & 4.38 & 4.9 & 5.22 & 5.7 & 5.92 \\
\hline 2 & 2.7 & 3.22 & 5.3 & 5.33 & 6.1 & 6.52 \\
\hline 3 & 1.35 & 1.93 & 5 & 5.31 & 6.5 & 7.03 \\
\hline 4 & 0 & 0.52 & 4.5 & 5.22 & 6.8 & 7.44 \\
\hline
\end{tabular}

Table 1: Output Power of Cascaded EDFAs

\begin{tabular}{|c|c|c|c|c|c|c|}
\hline Amplifier & 1550 Exp & $\begin{array}{c}1550 \\
\text { Model }\end{array}$ & 1554 Exp & $\begin{array}{c}1554 \\
\text { Model }\end{array}$ & 1558 Exp & $\begin{array}{c}1558 \\
\text { Model }\end{array}$ \\
\hline 1 & 5.1 & 5.14 & 5.5 & 5.61 & 4.6 & 4.9 \\
\hline 2 & 3.9 & 4.09 & 5.7 & 5.79 & 5.3 & 5.61 \\
\hline 3 & 2.5 & 2.92 & 5.4 & 5.86 & 6 & 6.22 \\
\hline 4 & 1.4 & 1.63 & 5.1 & 5.7 & 6.3 & 6.72 \\
\hline
\end{tabular}

Table 2: Second Run, Output Power of Cascaded EDEAs 


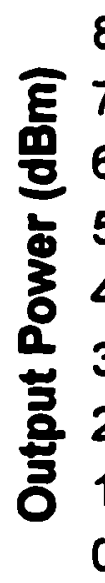

8
7
6
5
4
3
2
1
0

3
1
1
1

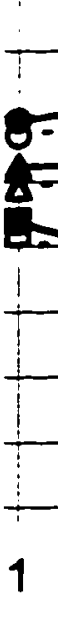

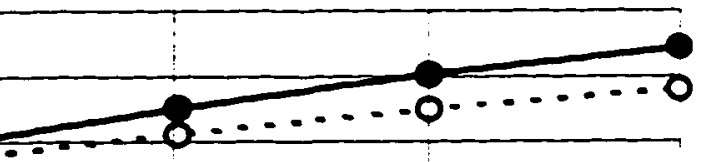
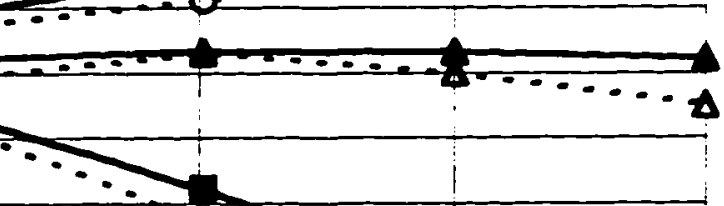

$+2$

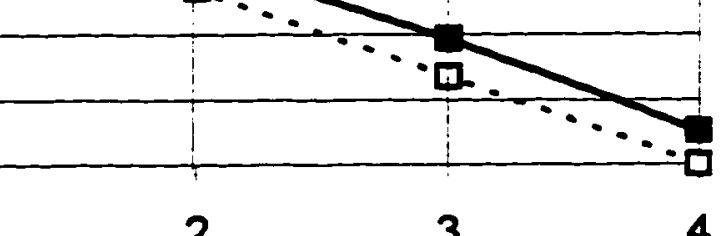

2

3

No. of Amplifiers

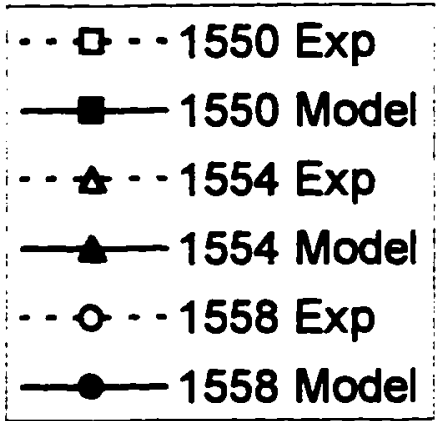

1558 Model

Figure 12: Four Cascaded EDFAs
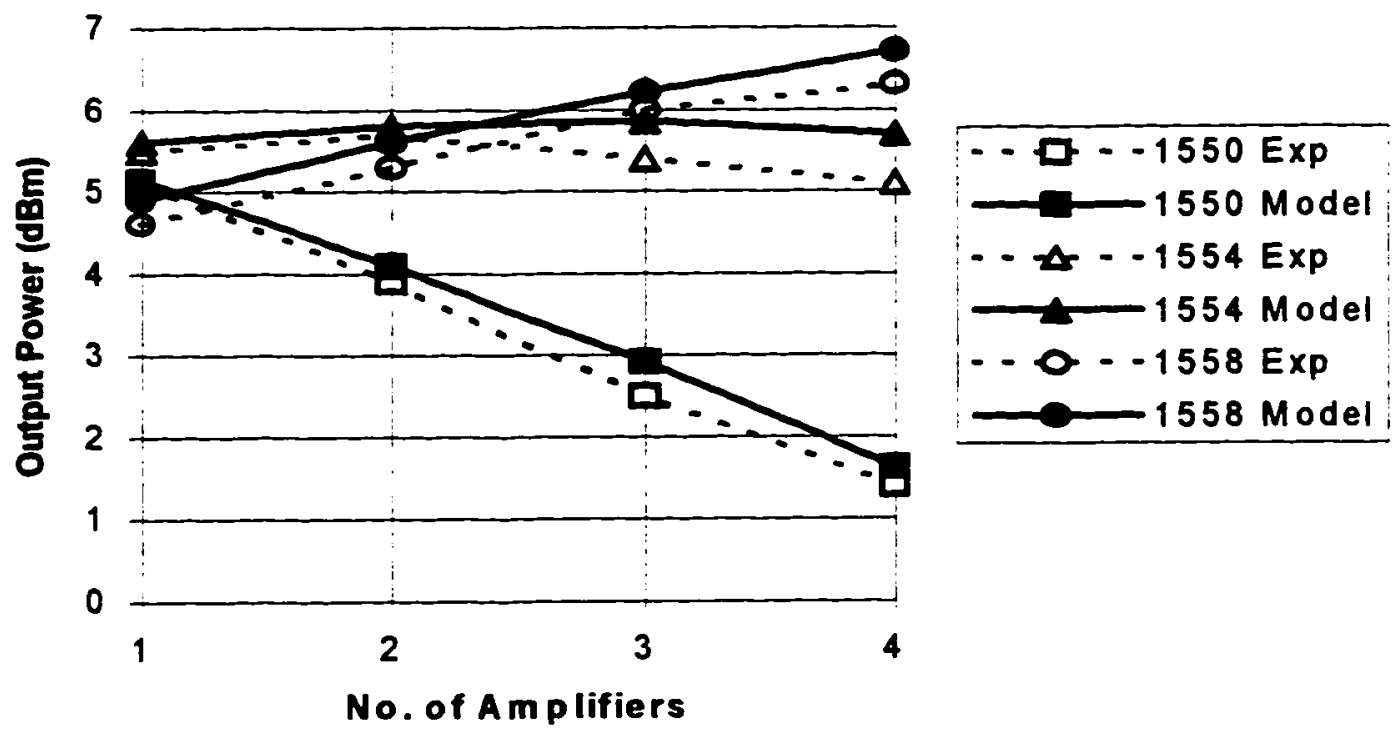

Figure 13: Second Data Set for Cascade

Figure 12 and Figure 13 show that the gain can be accurately predicted for several cascaded EDFAs. This 
results from the fact that the model is independent of the input powers of EDFA. As the specific wavelength's powers change, the model is capable of adapting to the given inputs. 


\section{Chapter 5}

Explanation of the Convergence and Divergence Points

The following is an attempt to describe how the phenomenon previously described occurs. This is not a theory, per se, but more of a first look into a possible explanation of the results. The logic behind predicting gain tilt begins with the basic laser equations and absorption and emission spectrums. The extent of absorption and the likelihood of emission of photons in a lasing medium controls the degree of tilt.

The two level laser rate equation is: ${ }^{13}$

$$
\frac{\partial N_{1}}{\partial}=-\frac{\partial N_{2}}{\partial t}=\left(n_{2}-\frac{g_{2}}{g_{1}} n_{1}\right) c \phi \sigma_{21}+\frac{n_{2}}{\tau_{21}}-W_{p} n_{1}
$$

for steady state where the rate of change of electrons in the $\mathrm{N}_{1}$ and $\mathrm{N}_{2}$ energy levels is equal to 0 . The $\mathrm{N}_{1}$ level is the ground state and the $\mathrm{N}_{2}$ level is the first excited state as seen in Figure 3. The small case ' $n$ ' is the electron density in the $N_{1}$ level and the $N_{2}$ level, $\phi$ is the photon density and $\sigma_{21}$ is the stimulated emission cross section. W is the effective pumping rate and $n_{2} / \tau_{21}$ is the spontaneous emission rate. The ratio $\mathrm{g} 2 / \mathrm{g} 1$ is a relation of the cross section of the emission and absorption spectra and is given by : 


$$
\sigma_{12}=\frac{g_{2}}{g_{1}} \sigma_{21}
$$

where $\sigma_{::}$is the absorption cross section and $\sigma_{::}$is the emission cross section."i Both cross sections are wavelength dependent.

Equation 8 is the conservation of energy. The energy used to raise electrons to the excited state equals the energy produced by the spontaneous and stimulated emissions resulting from electrons which return to the ground state. Another condition for predicting gain tilt is that the amplifier is saturated. The output signal power is close to all of the available output power of the EDFA. From the discussion on EDFA operation, an amplifier in saturation has a large number of signal photons compared to noise photons. As explained in the introduction to EDFAs, the lasing medium is capable of absorbing or emitting photons in the $1550 \mathrm{~nm}$ spectral region. A photon that is produced through stimulated emission that is absorbed later in the medium is considered energy that has been reabsorbed. Reabsorption is based on probability. There is a probability that an electron in the ground state is encountered by a photon and a probability that the photon energy is sufficient to raise the ground state electron to the exited state. The absorption cross section is related to the probability as: 


$$
\sigma_{12} \nsim P_{\mathrm{mt}} P_{\mathrm{en}}(\lambda) L
$$

where $P_{::}$is the probability per unit length of encountering an electron in the ground state, $P_{e n}$ is the probability that the electron encountered can be excited to the $\mathrm{N}$ : level by the energy of the photon, and $L$ is the length of the doped fiber.

The required energy to excite an electron is not a simple single energy threshold, but a time dependent threshold. This results from the stark splitting of the excited state of the Erbium atom. At any specific time, the energy required to excite an electron is dependent on the phonon interaction and local crystal defects. It stands to reason that shorter wavelength photons, those with higher energies, would be more likely to be reabsorbed by the medium than ones with longer wavelengths. The higher energy photon has a larger portion of the energy level to which it can elevate an electron.

It can be assumed that within the excited state level there is a distribution of electrons that is unaffected by the presence of a pump laser in the operating band of the amplifier. This follows from the condition that an EDFA can be reasonably modeled as a 2 level system even though it is really a 3 level system. The electrons excited by the pump laser to level $\mathrm{N}_{3}$, decay to the $\mathrm{N}_{\text {: }}$ excited state in tens of 
microseconds. At the $\mathrm{N}$ : level, the electrons distribute themselves to the available energy levels decaying in tens to hundreds of milliseconds. The $N_{3}$ level decay time is several orders of magnitude smaller than that of $\mathrm{N}_{2}$. This implies that the $\mathrm{N}_{3}$ level can be ignored and the gain medium becomes a two level system.

An electron excited from a laser with a wavelength in the amplification band of the EDFA (1530-1565 nm) moves from the ground state to the $\mathrm{N}_{\text {: }}$ level without needing to pass through the $N_{3}$ energy level. Since the $N_{3}$ level is ignored, the distribution of electrons in the excited state should equalize its self whether there is operating band wavelength reabsorption or not. The emission probabilities should not change given that the excited state maintains its typical distribution. The probability that a photon encounters an excited electron should not noticeably change, implying that the emission cross section should remain constant.

In steady state, a number of short wavelength signal photons, kl, are absorbed. The excited electrons evenly distribute themselves throughout the excited level due to the effect of thermalization.: Similarly, a number of long wavelength signal photons, $k 2$, are absorbed and the electrons are distributed throughout the energy level. From 
the absorption spectrum above $k 2<\mathrm{kl}$, and thus more short wavelength photons should be absorbed.

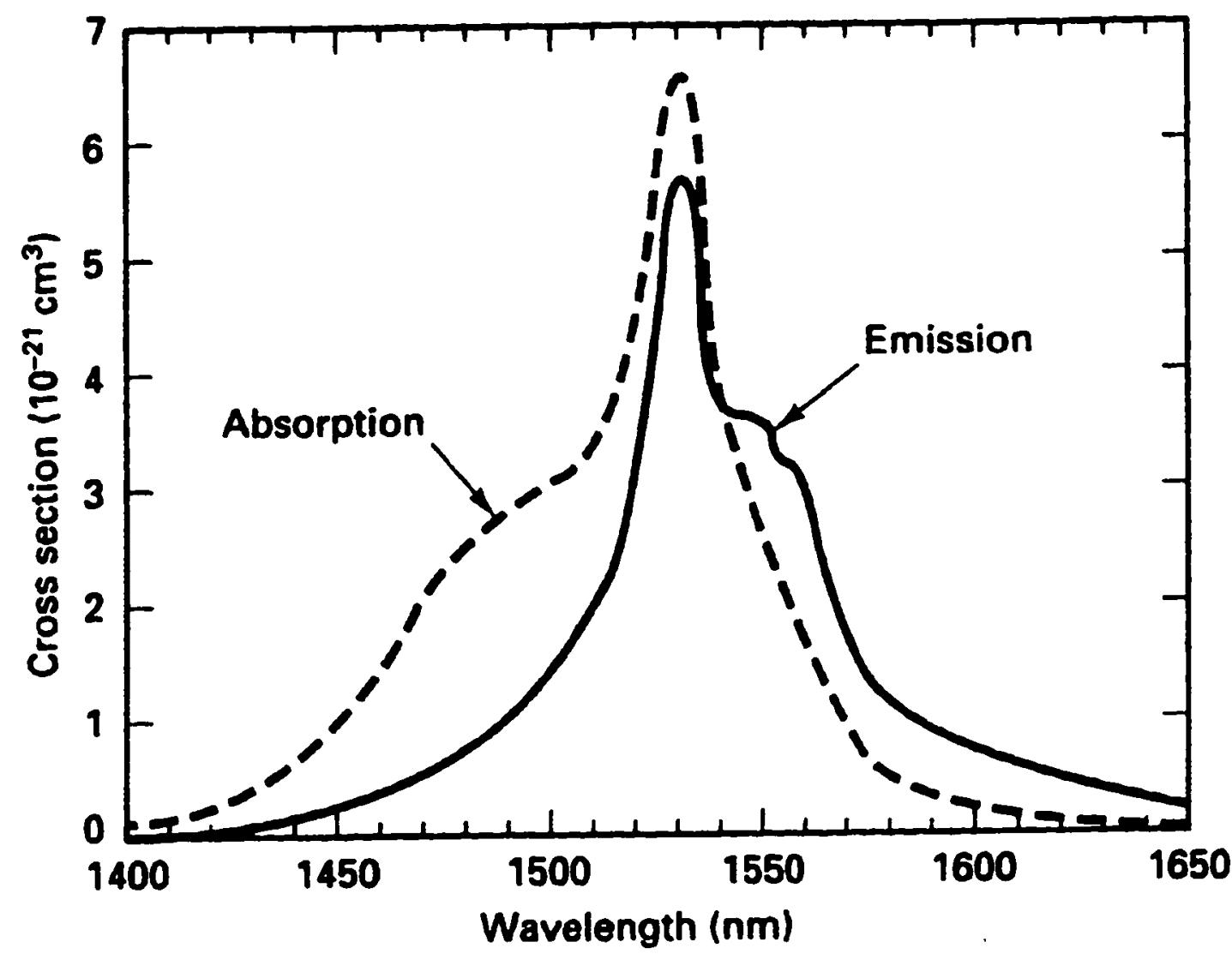

Figure 14: Absorption and Emission Cross Section ${ }^{16}$

The ratio of absorption to emission curve shows that the shorter wavelengths, $\lambda_{2}$, should be attenuated somewhat under the above conditions. The output power of the signal will be reduced and thus the gain of that signal will be reduced. A longer wavelength, $\lambda_{i}$, signal will see a smaller reduction and benefit from the extra electrons provided to the excited level by the shorter wavelength. Therefore, for 


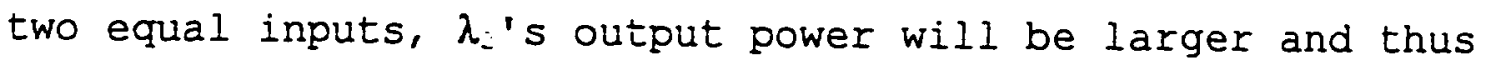
its gain will be larger than the shorter wavelength.

The output power of the EDFA itself, in saturation, is constant. The total input power to the EDFA exactly determines the gain. The total gain curve of an EDFA and the gain curve of a single wavelength are the same. This assumes that the source noise is negligible. The equivalency is a result of the definition of total gain. The total gain, in $d B$, of the EDFA is the total output power less the total input power, or in watts, the total gain is the sum of the inputs divided into the sum of the outputs. When only a single wavelength is present, the two gain curves are exactly the same. In saturation, the total output power is a constant, and therefore the total gain of the amplifier is the difference ( $d B)$ or quotient (W) between this constant and the total input power.

With the addition of other wavelengths, the total gain curve remains unchanged while the individual wavelengths' gain curves change. The total gain curve does not change since it is related to the total number of available electrons in the excited state. The first wavelength introduced follows the total gain curve. If the power of the first wavelength is set to saturated the amplifier, a specific gain results. As will be explained below, the 
introduction of a second wavelength causes the gain of the first wavelength to change. If the second signal is very weak compared to the first, then the effect on the gain will be minimal. If the two are comparable, then the effect should be larger. If the second is larger than the first, the effect should be larger yet.

It has been determined that a longer wavelength will achieve a greater gain from an EDFA than a shorter, copropagating wavelength. The amount is still to be discussed, but if the shorter wavelength reduces in gain from the amplifier's total gain, and the total gain of the EDFA doesn't change, the longer wavelength should have more gain than the total gain of the EDFA at the same total input power.

$$
G_{1}+G_{2}=G_{\text {total }}
$$

The point at which a shorter wavelength's gain leaves the total gain curve due to the introduction of a second signal is defined as the divergence point ( Figure 7). The path after this is still unknown, but from logic it must exist. The gain curve of the individual wavelengths is the gain of the wavelength vs. the total input power to the EDFA.

For the discussion on predicting gain tilt, it must be assumed that the emission spectrum is flat in the region of 
interest. The emission spectrum of Figure 14 above is relatively flat and the emission spectrum of the test amplifiers is more flat. This flat emission spectrum implies that an equal number of electrons, in steady state, are at all wavelengths.

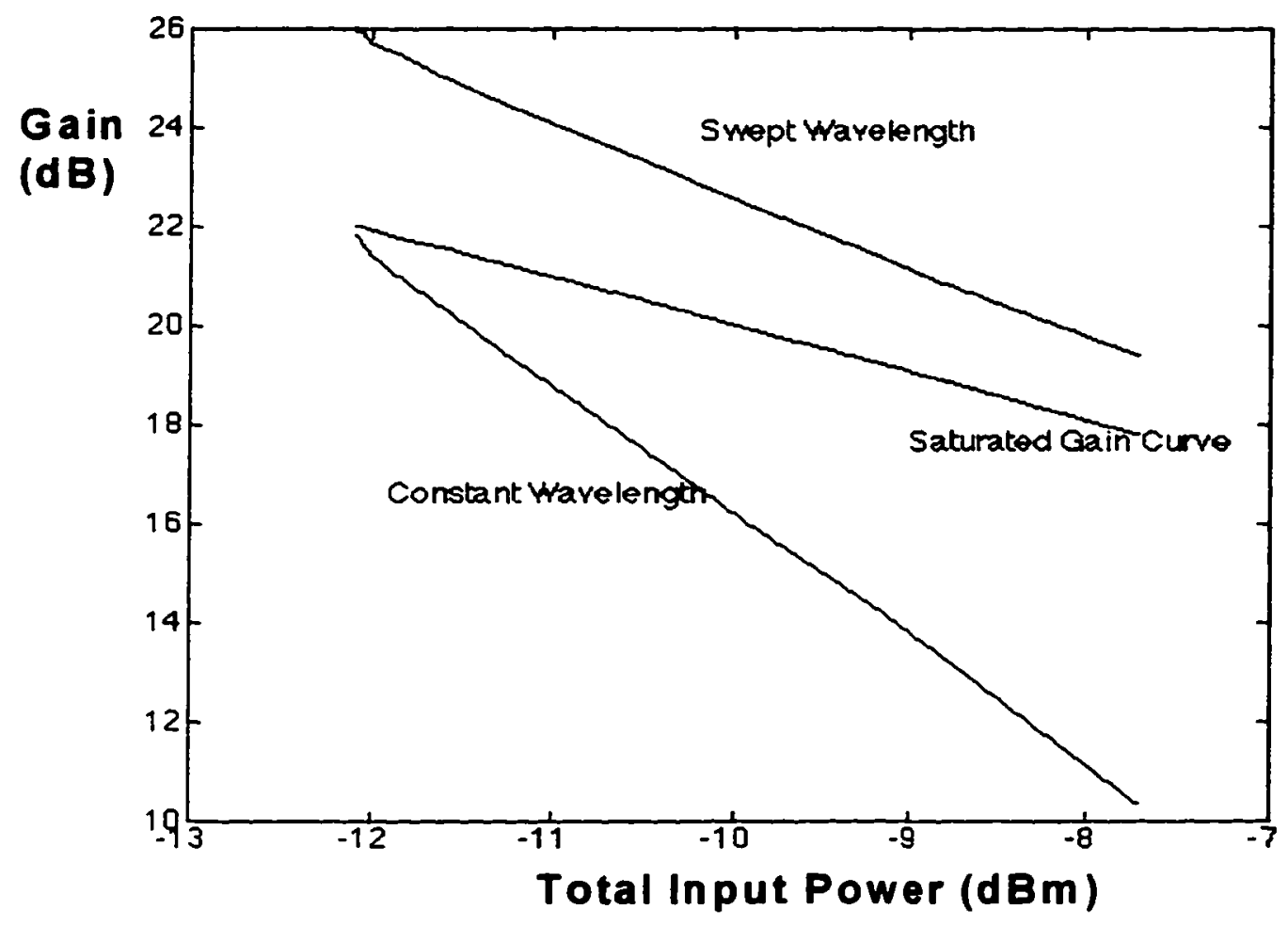

Figure 15: Divergence of Gain

The path which the gain curve takes in getting from the divergence point to the gain produced with a second wavelength present should be linear. Experiments show that the short wavelength gain curve for saturated EDFAs is linear, anchored at one end by the divergence point. This 
is reasonable since the EDFA total gain curve is linear in the saturated region. See Figure 15.

A line in a log-log graph is a power series in a linear-linear graph. For the total gain curve, a doubling of the input power results in halving the gain. A result of the avalanche gain effect reaching an asymptote. With greater numbers of photons present, each creates fewer photons at the end of the amplifier.

The number of photons present for the shorter wavelength is further decreased by reabsorption. These reabsorbed photons would be stimulated to emission by the signal in a single wavelength system. In a WDM system, the reabsorbed photon has an equal likelihood of being stimulated to emission by other wavelengths present. This results in a reduction of photons at the shorter wavelength. Less photons means a reduction in the initiation of stimulated emission, but the presence of other wavelengths means the amplifier still operates as a saturated amplifier. This suggests that the gain should still be a power series with a reduction in the exponent. Therefore the curve on the log-log graph will also be linear.

Comparing two different starting power levels for $\lambda$ : brings about another useful point. The signal with greater initial power will drive the EDEA further into saturation. 
There will be more electrons in the ground state at any one instant in time. More photons will be reabsorbed. With $\lambda$ : present, gain tilt will result.

The length of the doped fiber needs to be considered. The photon amplification process is an avalanche process. For any point in the doped fiber the photons coming in will produce more photons going out unless the section is saturated. A saturated EDFA has only a portion of the end of its doped fiber saturated. The beginning of the fiber is acting as an unsaturated amplifier. This is a result of the optical power at the beginning of the fiber not being sufficient to saturate it, only with amplification does the signal have sufficient power to saturate a portion of the fiber. As the total input power to the amplifier increases, more of the doped fiber will be saturated.

Returning to the discussion on gain, the amount of gain tilt will be different for the two starting powers $\lambda_{1}$. For the case of the $\lambda$ : with less power, matching the power of the $\lambda_{\text {: }}$ with the $\lambda_{2}$ 's power results in a doubling of the total input power. More input power leads to greater saturation of the EDFA and a greater probability of photon reabsorption. Within the EDFA's doped fiber, there will be a certain length of saturated fiber. 
For the higher power short wavelength case, matching the $\lambda_{i}^{\prime}$ s power to the $\lambda_{:}^{\prime} s$ power also increases the saturation of the EDFA. More importantly, it increases the amount of the doped fiber which is saturated. The length traveled along the doped fiber before reaching the point of saturation is less than the previous case. Therefore the probability of encountering an electron in the ground state increases and the active length of the doped fiber is greater.

From Equation 10, the probability of encountering a ground state electron $\left(P_{i n c}\right)$ is increased with increasing input power. This is a result of increased saturation of the EDFA. The doped fiber itself becomes saturated sooner for a larger starting input power. If significant reabsorption is considered to only occur within saturated doped fiber, then the value of $L$ in Equation 10 will increase for the large starting power case. This will result in a greater absorption coefficient for the same wavelength.

The extra reabsorption causes an additional decrease in gain for an equivalent increase in total input power. The gain curve has already been shown to diverge from the total gain curve at the power of the shorter wavelength. The reduction in gain for the greater power short wavelength as 
compared to the weaker case indicates that the former will have a steeper slope of its gain curve. The steepness of the slope increases as the starting input power increases for the short wavelength. The two gain curves should intersect at some point since their slopes are different.

All of the gain curves should intersect. The intersection points of the curves have two possible results. Any pair of curves could intersect at a distinct point. The location of these distinct points could then be determined by a equation. The other result is that the all curves could intersect at one single point. Experimentation shows that the latter is the case. This point will be defined as the convergence point.

The total gain curve of an EDEA never changes. The divergence point, from logic is easily definable for any two wavelengths present. The convergence point is shown through experimentation. This suggest that all that is required is an equation that fits the two data points and follows the diverging gain curve. The second wavelength's output would be easy to determine. Since the two wavelengths share the total output power, the longer wavelength would simply receive the remainder of the power available.

Once the equation for the calculation of gain for the shortest wavelength is known, the gain may be calculated by 
simply knowing the total input power and the power of the wavelength in question. The second wavelength's gain is then calculated by subtracting the first signal's output power from the maximum output power of the amplifier. The power that remains is the combination of the ASE and the other signal power. Since the model only considers the saturated region of the amplifier, the ASE can be assumed to be negligible. Therefore the remaining power is all in the other signal and the gain is determined by dividing the output by the input.

This method can be expanded to include more than two wavelengths. If a third wavelength were present, the above discussions would still apply. The shortest wavelength's gain could be determined from the previous paragraph by first ignoring the third, and longest, wavelength. Increasing the third wavelengths power will not improve the gain of the shortest wavelength since the total power must now be shared with three signals. The longer wavelength should also rob power from the shorter wavelength through the power reabsorption and redistribution. The divergence concept applies to the addition of the third wavelength. From the convergence point argument, the shortest wavelength ought to have another convergence point for this configuration. 
Once the first wavelengths gain is determined the second wavelength would usually receive the remainder of the power in the EDFA. The third wavelength must share power with the second wavelength. Since the third wavelength is longer, the second wavelength's gain will be reduced. The only crosstalk effect in an EDFA is power sharing which implies that the shorter wavelength signal only reduces the available power for the other wavelengths. In effect, the EDEA for the two longer wavelengths is an EDFA with less power.

To complete the discussion, the above theory should still predict that the gain of any signal wavelength by itself should equal the gain curve of the amplifier. The reabsorbed photons of the signal wavelength raise electrons to the excited state. In normal saturated operation, the signal photons outnumber the noise photons considerably. Therefore a majority of the excited electrons from reabsorption would be stimulated to emission by the signal wavelength, returning the energy absorbed back to the signal wavelength where it came from. The gain limit is then the amplifier gain curve since the output power of the EDFA is Iimited and the amplifier is operated in the saturated region. 


\section{Chapter 6 \\ Conclusion}

A method for calculating the EDFA gain for multiple signals in a WDM optical environment has been demonstrated. Accurate parameters are achieved using a single type of experiment. The data is used to produce a model that calculates the gain for any combination of signals with any combination of input powers as long as the EDFA is saturated. This method then allows for link evaluation as signal and noise levels can be calculated from the gain.

The experimental results are repeatable suggesting that the next step is to expand upon the theory behind the observations. The explanation given in this thesis is a starting point for future work. Greater investigation of the absorption and emission characteristics of the doped fiber is in order to be able to predict the convergence point. With the ability to predict the convergence point, the need for empirical testing will be avoided.

With the expanded understanding of the doped fiber characteristics, the model should be expanded to include the unsaturated region of the amplifier. A comparison between the gain tilt effect in the saturated and unsaturated regions of the EDEA will give insight into the strength of the model concepts. 


\section{Notes}

: Desurvire, Emmanuel. Erbium-Doped Fiber Amplifiers: Principles and Applications. New York: John Wiley and Sons, Inc., 1994. 17-25.

: Desurvire 17-25.

I Green, Paul E. Fiber Optic Networks. Englewood Cliffs, New Jersey: Prentice-Hall, Inc., 1993. 242.

; Desurvire 20.

Desurvire 209 .

Desurvire 9.

Desurvire 225.

Desurvire 225.

"Henry, Paul S. "Lightwave Primer." IEEE Journal of Quantum Electronics QE-21 (1985), 1873.

$\therefore$ Green 235 .

$\because$ Agrawal, Govind P. Fiber-Optic Communication Systems. New York: John Wiley and Sons, Inc., 1992. 334.

$:=$ Agrawal 375 .

:3 Koechner, Walter. Solid-State Laser Engineering. Springer Series in Optical Sciences 1. New York: Springer-Verlag, 1976. 27 .

$: 4$ Koechner 5 .

:- Desurvire 8 .

$\therefore$ Green 242 . 
Appendix A

List of Acronyms

WDM

EDFA

ASE

$\mathrm{ER}^{3+}$

BER

OSA
Wavelength Division Multiplexing

Erbium Doped Fiber Amplifier

Amplified Spontaneous Emission

Erbium Ion

Bit Error Rate

Optical Spectrum Analyzer 


\section{Appendix B \\ Matlab Program for Model}

$81,2,3$ refer to the shortest to longest wavelength present \&respectively

Totout $=10$

pwr1 $=-13 ;$

pwr2 $=-13 ;$

pwr3 $=-13$;

pointgain12 = 10 ;

pointpwr12 $=5$;

pointgain13 = 8 ;

pointpwr13 = 4 ;

pointgain23 = 18;

pointpwr23 $=6$;
sTotal output power of EDFA in $\mathrm{dBm}$ \&Input power of 3 wavelengths

zConvergence points obtained from table opoint corresponding to first owavelength, two present \& point corresponding to first owavelength, 3 present froint corresponding to second ofwavelength, 3 present

totpwr2 = logadder (pwr1, pwr2);

\&logadder adds 2 numbers in $d B$ totpwr3 = logadder (totpwr2, pwr3);

constart $=$ totout - pwr1; $\quad$ stotal gain curve of EDFA $m=$ (pointgain12 - constant)/(pointpwr12 - pwr1);

$\mathrm{yb}=$ constant $-\mathrm{m}^{\star} \mathrm{pwr} 1$

scalc slope of gain curve

gainla $=m^{*}$ totpwr2 $+y b$;

outla $=$ pwr $1+$ gainla; $\quad$ foain of 1,2 present

constant = gainla;

$m=($ pointgain13 - constant $) /($ pointpwr13 - totpwr 2$) ;$

$\mathrm{yb}=$ constant $-\mathrm{m}^{*}$ totpwr2;

scalc slope of gain curve

$\operatorname{gain} 1=m^{*} \operatorname{totpwr} 3+y b$;

out $1=$ pwr $1+$ gainl;

\&Gain of 1,3 present

zoutput of 1

constant $=10^{*} \log \left(10^{\wedge}(\right.$ totout/10)-10^(out1a/10) $)-$ pwr2;

qGain of 2 without others

$m=($ pointgain23 - constant $) /($ pointpwr23 - totpwr 2$) ;$

$\mathrm{yb}=$ constant $-\mathrm{m}^{\star}$ totpwr 2

\&Calc slope

gain2 $=m^{*} \operatorname{totpwr} 3+y b$;

out2 $=$ pwr2 + gain 2 ;

\&Gain of 2,3 present soutput of 2

out $3=10^{*} \log \left(10^{\wedge}(\right.$ totout $/ 10)-10^{\wedge}($ out $1 / 10)-10^{\wedge}($ out $\left.2 / 10)\right)$;

$\$ 3$ gets remainder of power 
Appendix C

Table of Convergence Points

Convergence point values for the 3 wavelengths used

\begin{tabular}{|c|c|c|c|c|c|c|}
\hline $\begin{array}{c}\text { Wavelengths } \\
\text { Present }\end{array}$ & $\begin{array}{c}1533 \\
\text { Gain }\end{array}$ & $\begin{array}{c}1533 \\
\text { Power }\end{array}$ & $\begin{array}{c}1550 \\
\text { Gain }\end{array}$ & $\begin{array}{c}1550 \\
\text { Power } \\
(\mathrm{dB})\end{array}$ & $\begin{array}{c}1558 \\
\text { Gain }\end{array}$ & $\begin{array}{c}1558 \\
\text { Power } \\
(\mathrm{dB})\end{array}$ \\
\hline $1533 / 1550$ & 12.5 & -7.5 & 18.0 & -7.0 & $\mathrm{~dB} / \mathrm{a}$ & $\mathrm{n} / \mathrm{a}$ \\
\hline $1533 / 1558$ & -2.0 & -3.0 & $\mathrm{n} / \mathrm{a}$ & $\mathrm{n} / \mathrm{a}$ & 13.0 & -3.4 \\
\hline $1550 / 1558$ & $\mathrm{n} / \mathrm{a}$ & $\mathrm{n} / \mathrm{a}$ & 12.7 & -5.3 & 13.5 & -3.6 \\
\hline $1533 / 1550 / 1558$ & -0.4 & -3.9 & 10.7 & -3.7 & 13.5 & -3.8 \\
\hline
\end{tabular}


Appendix D

LabVIEW Data Collection Program

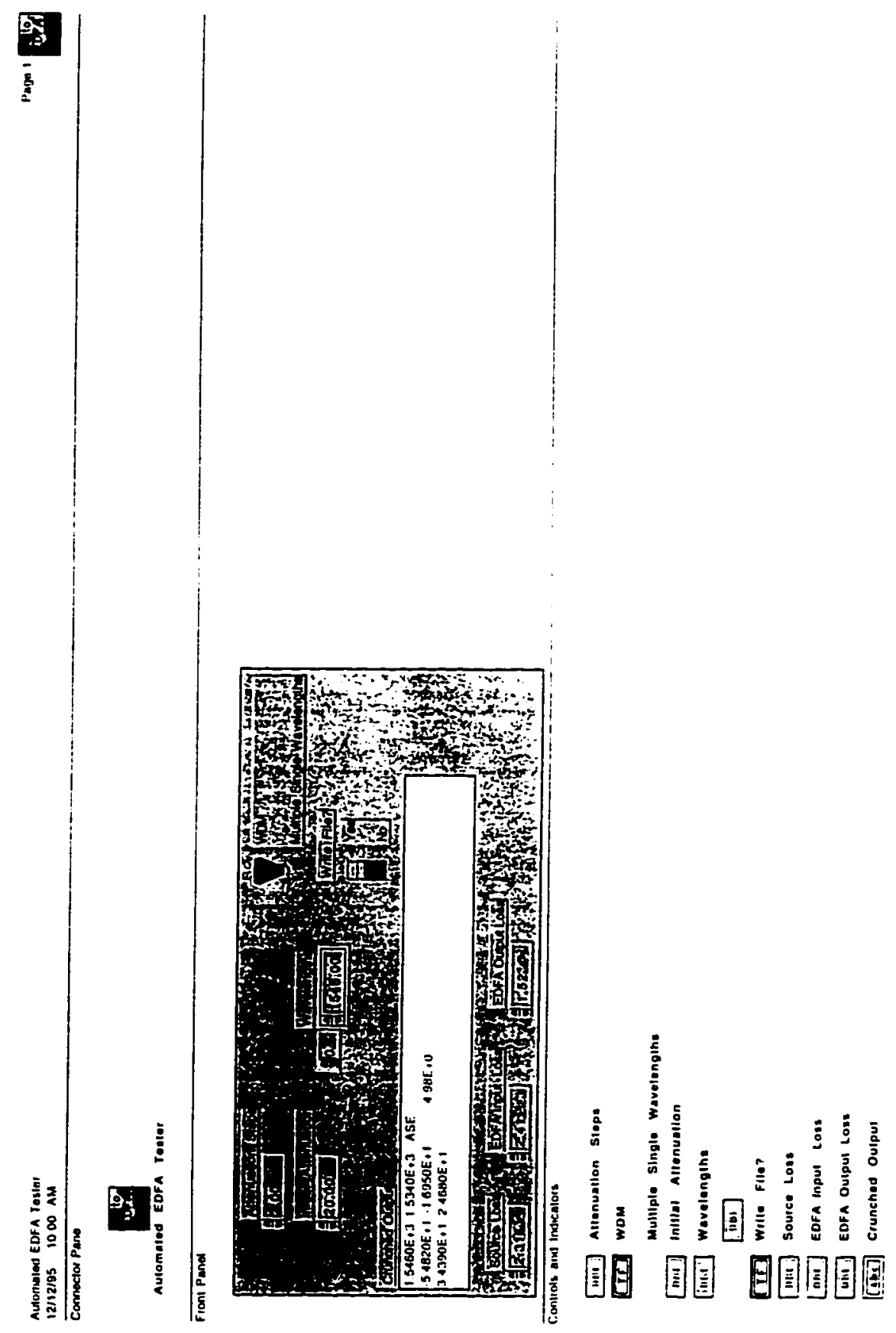




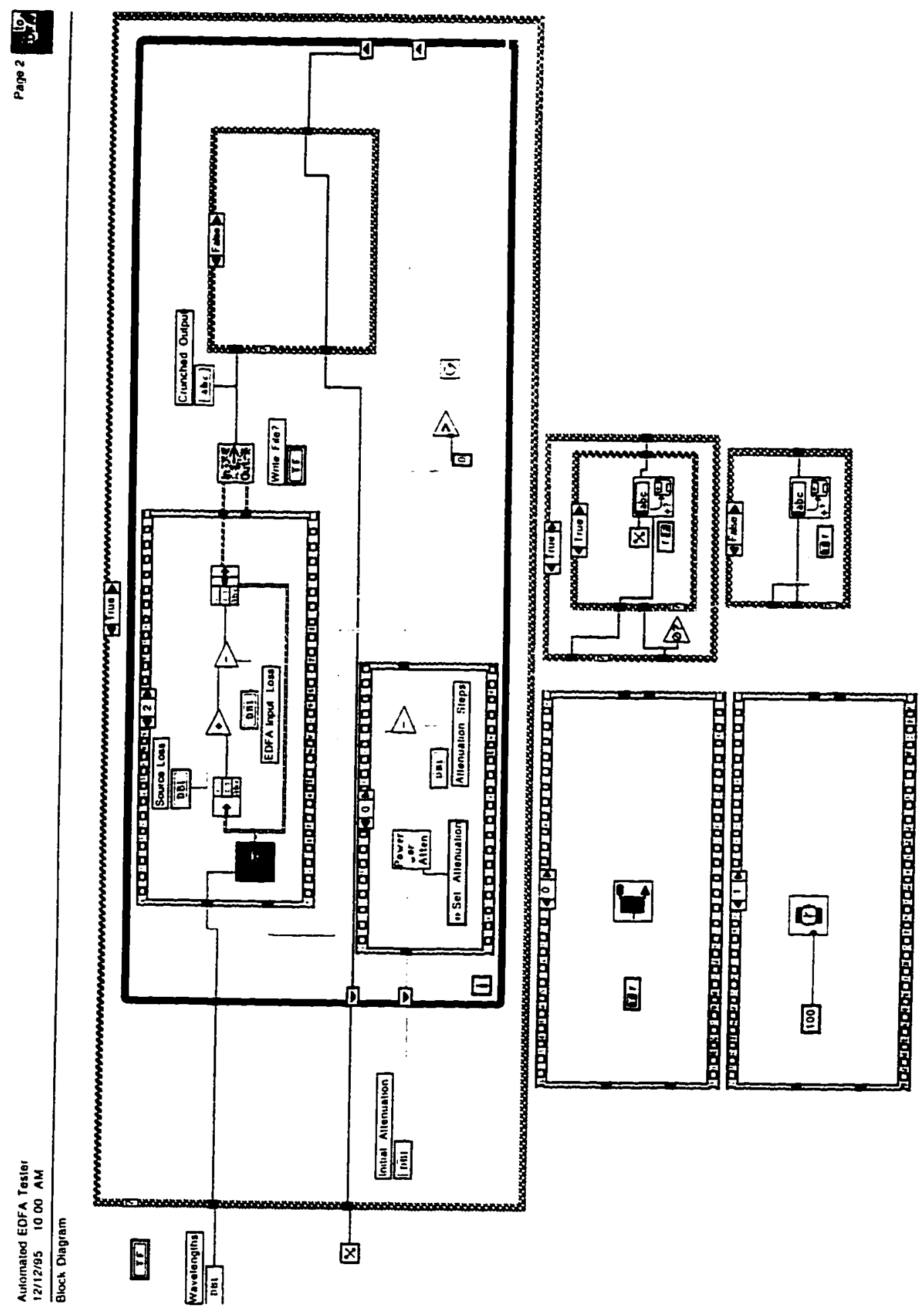




\section{䧑}

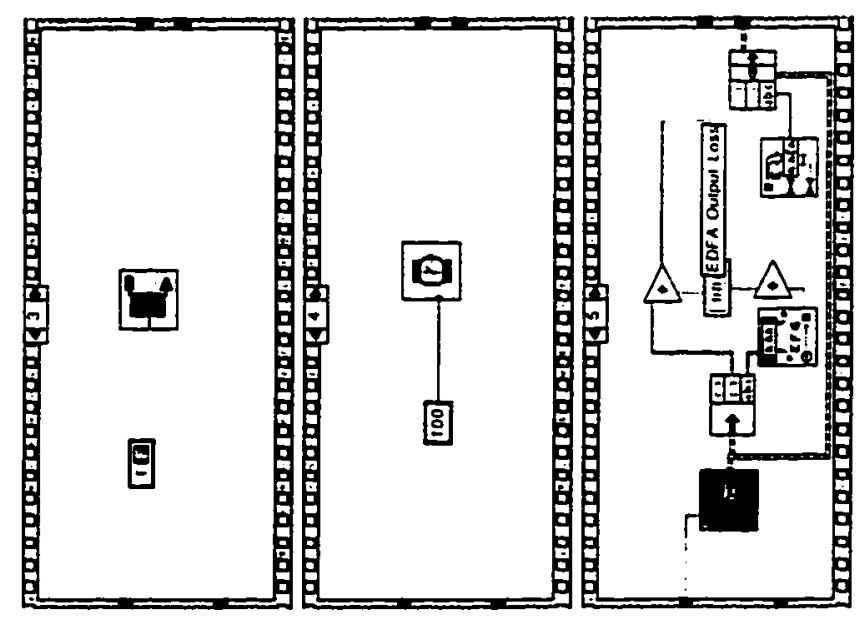

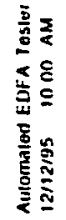




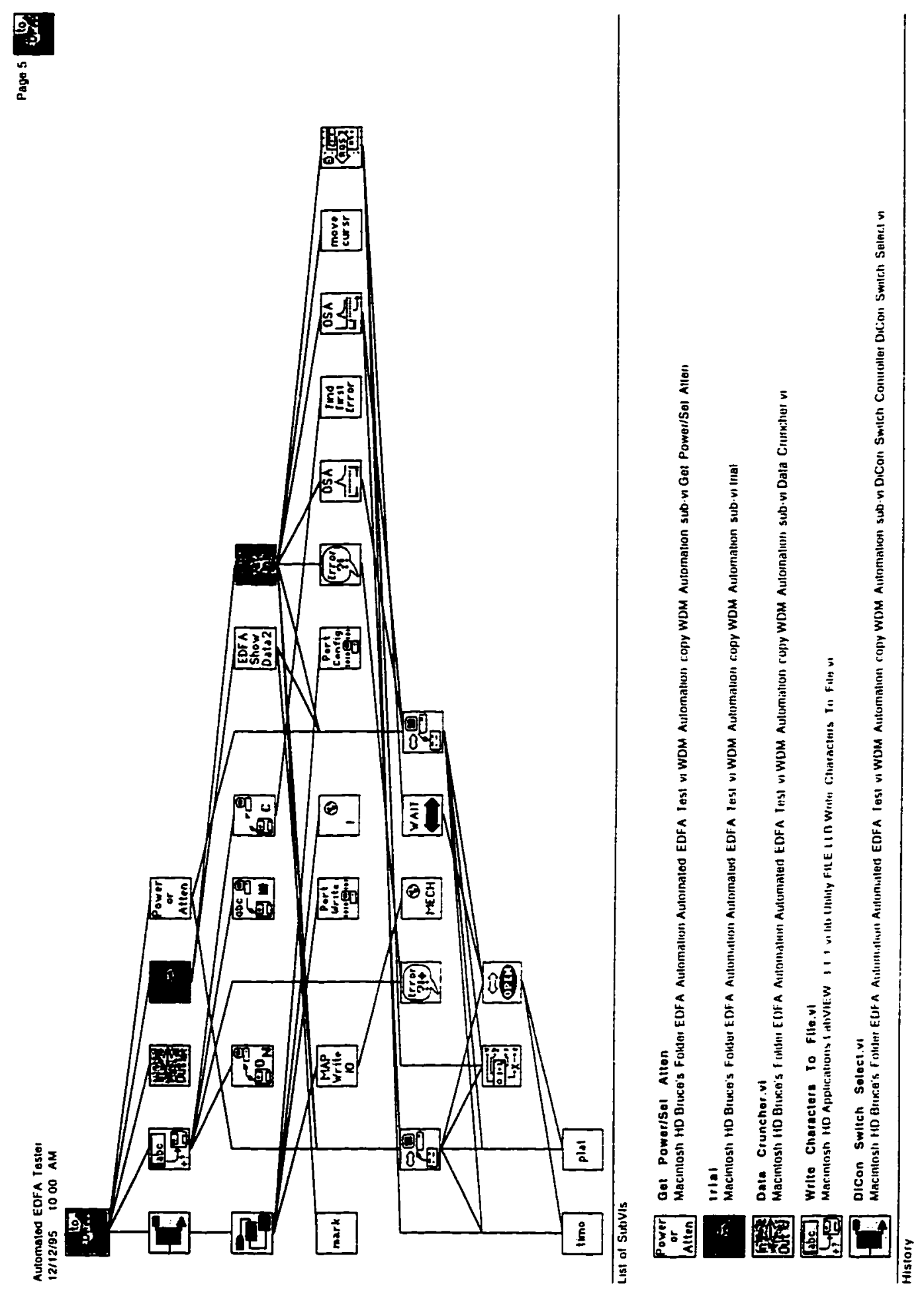


Appendix E

LabVIEW Model of EDFA .

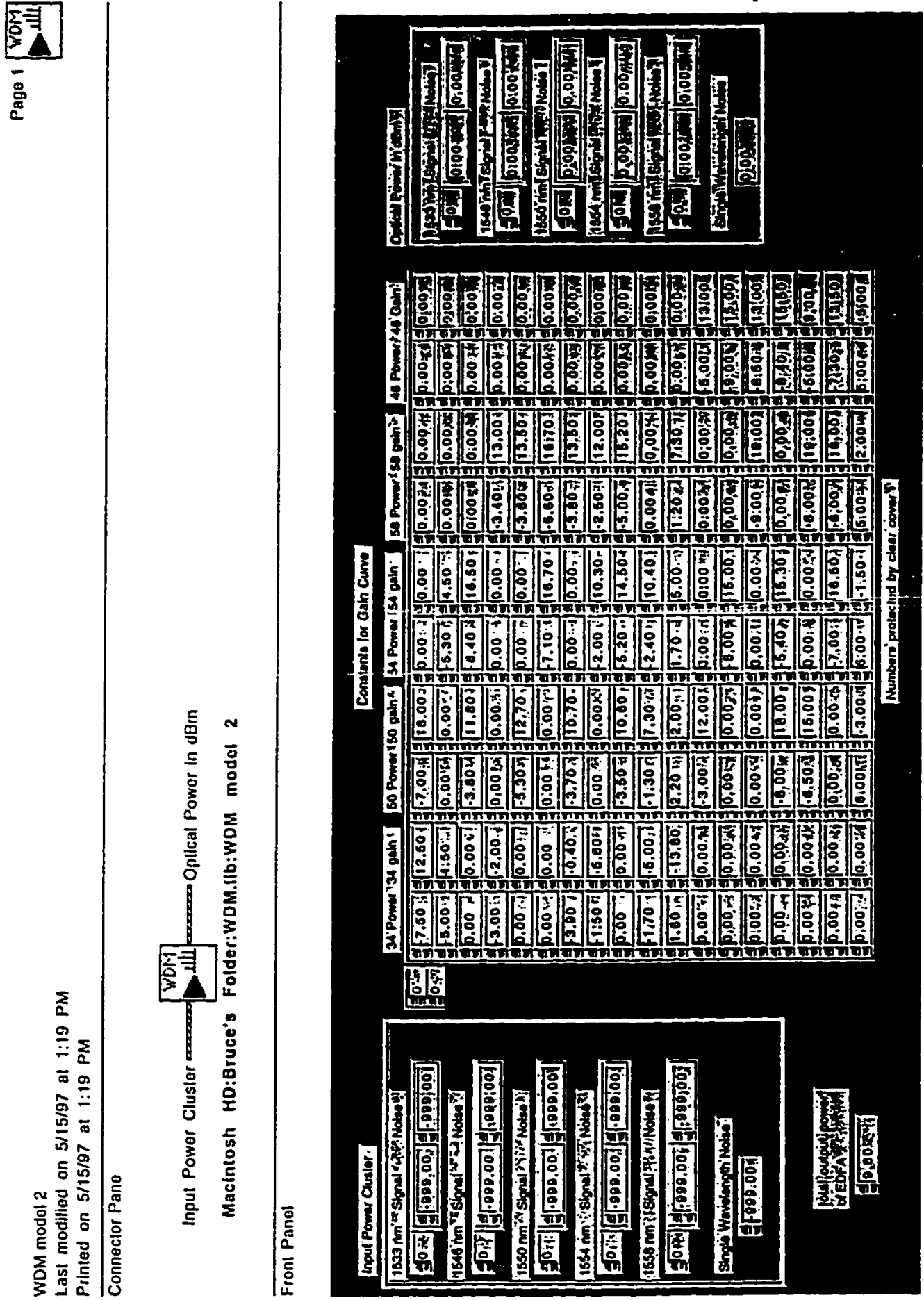




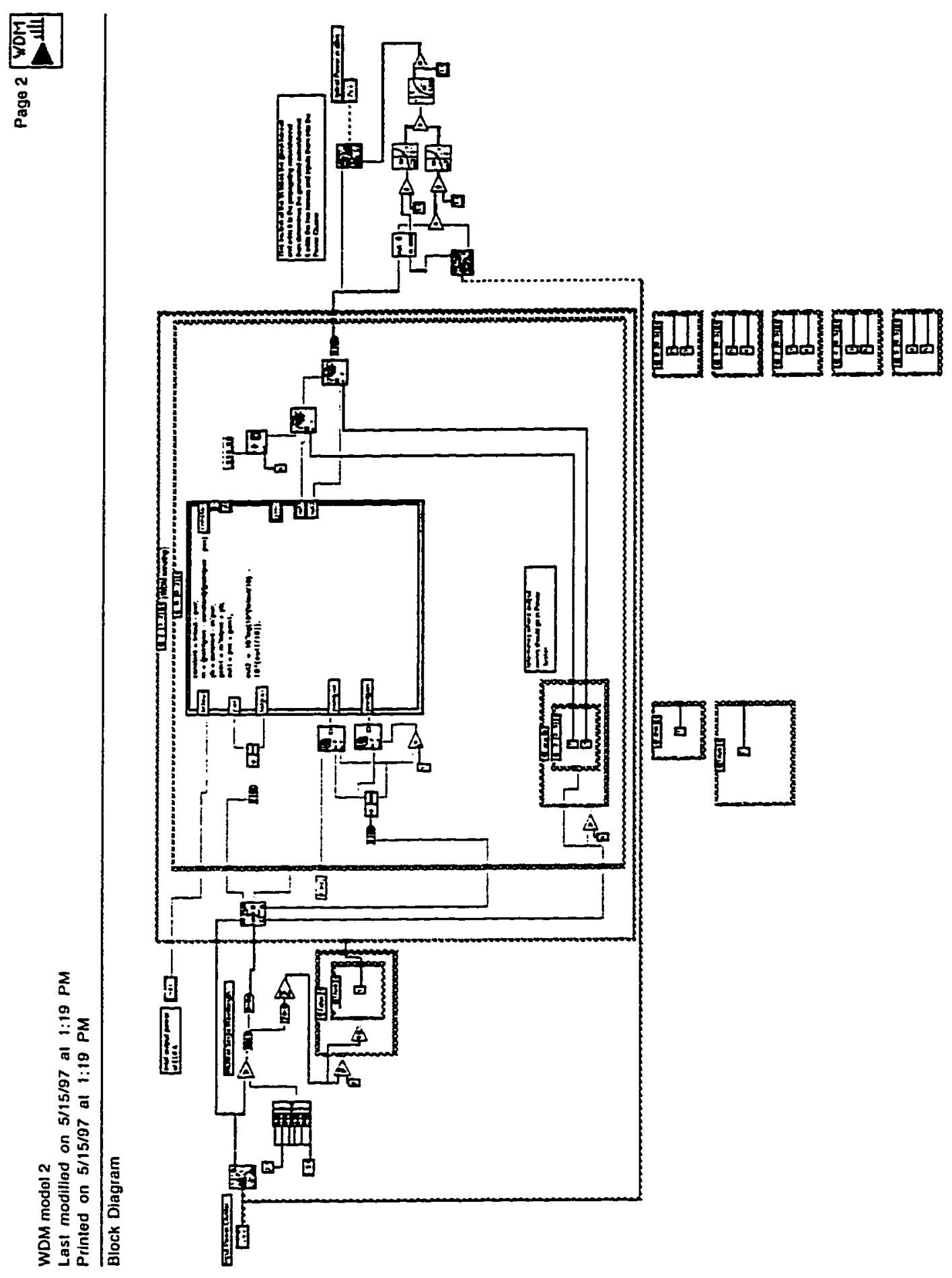



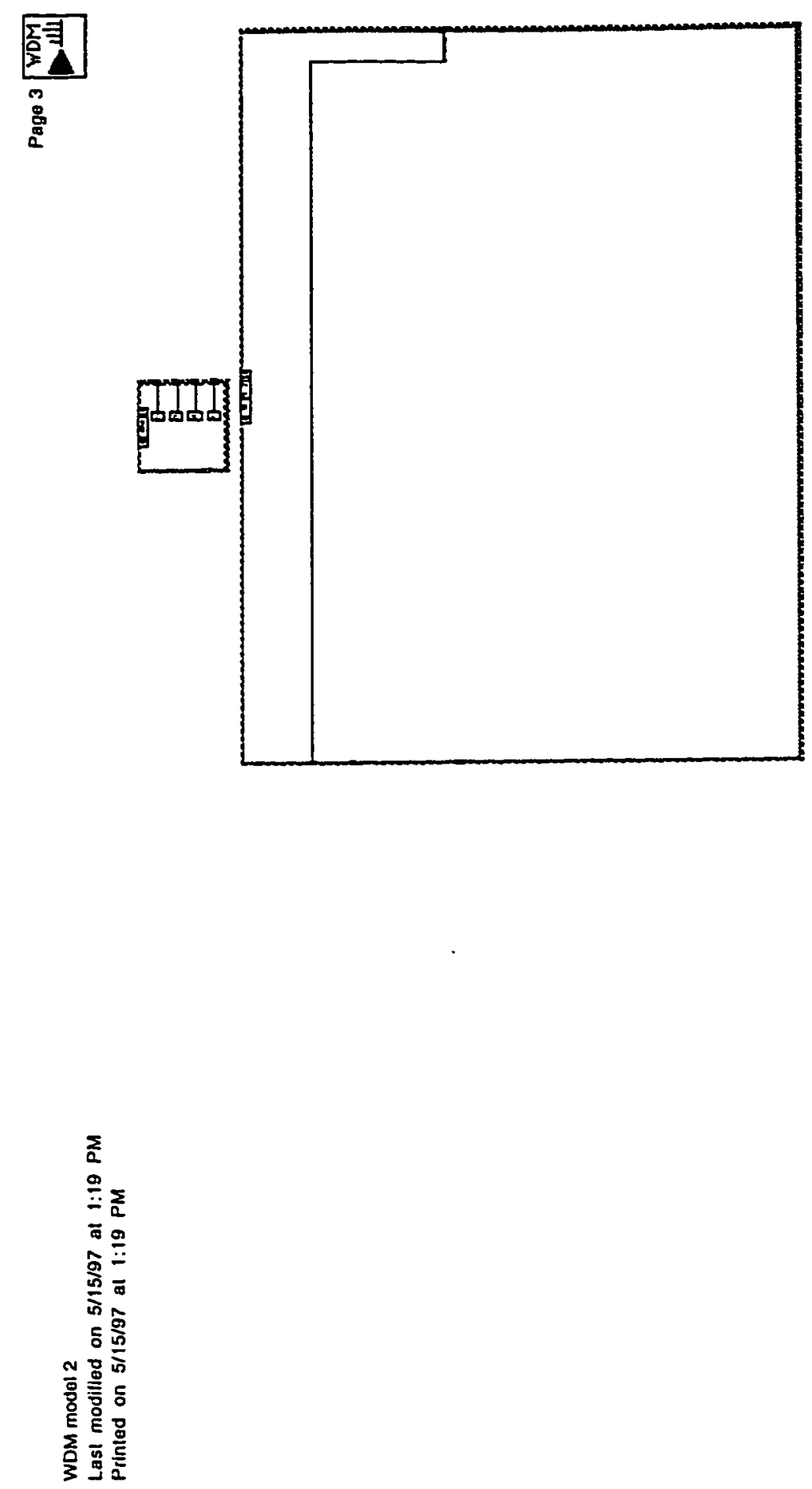


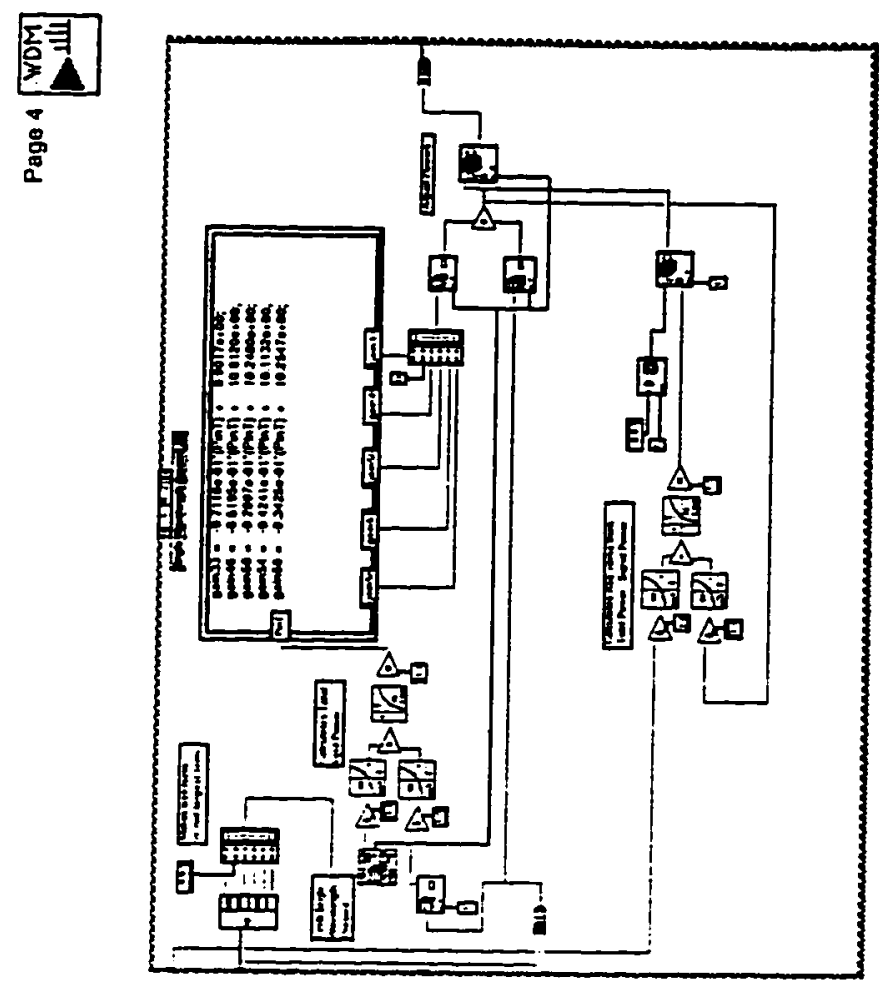

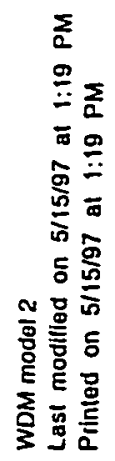



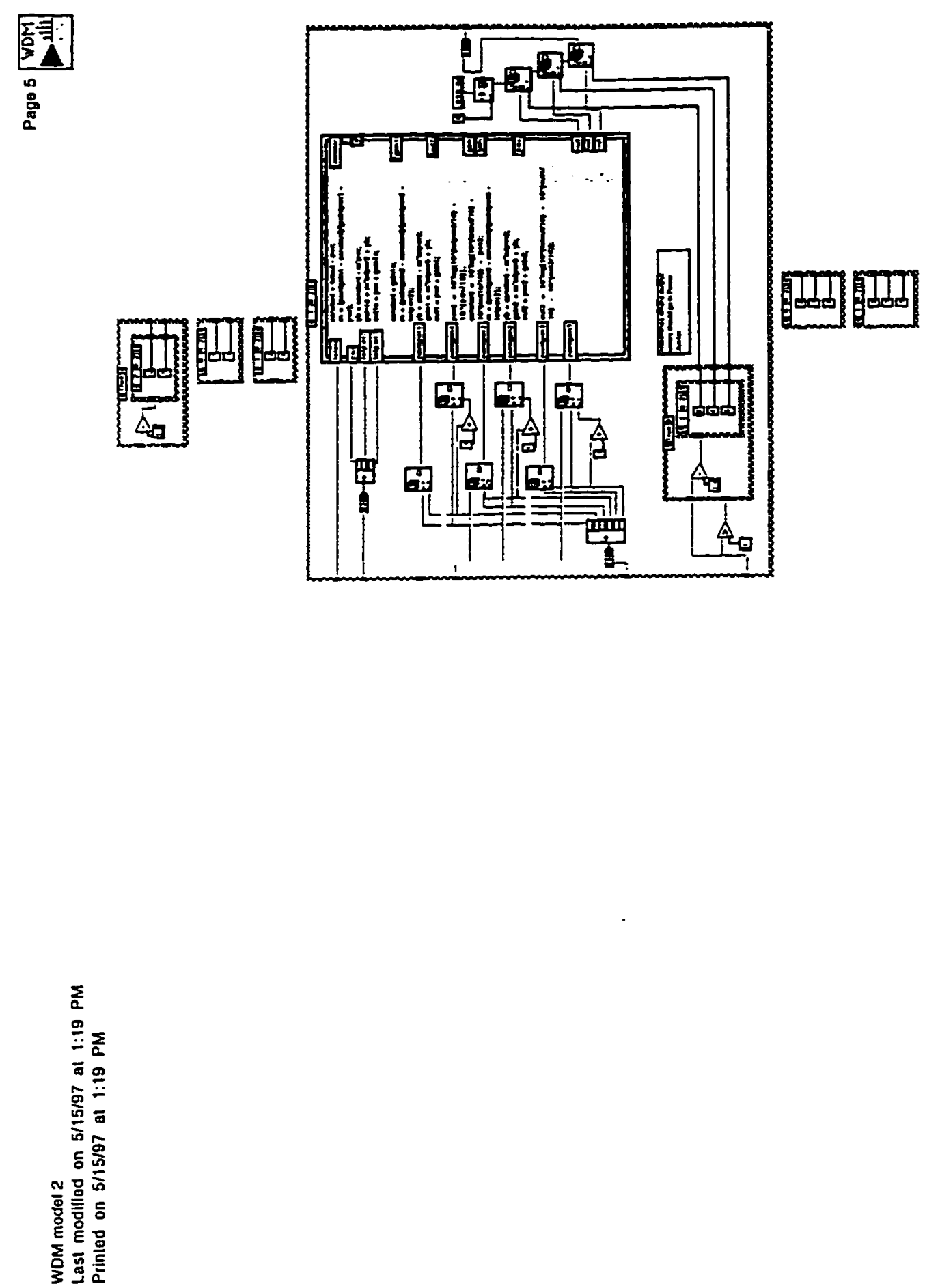


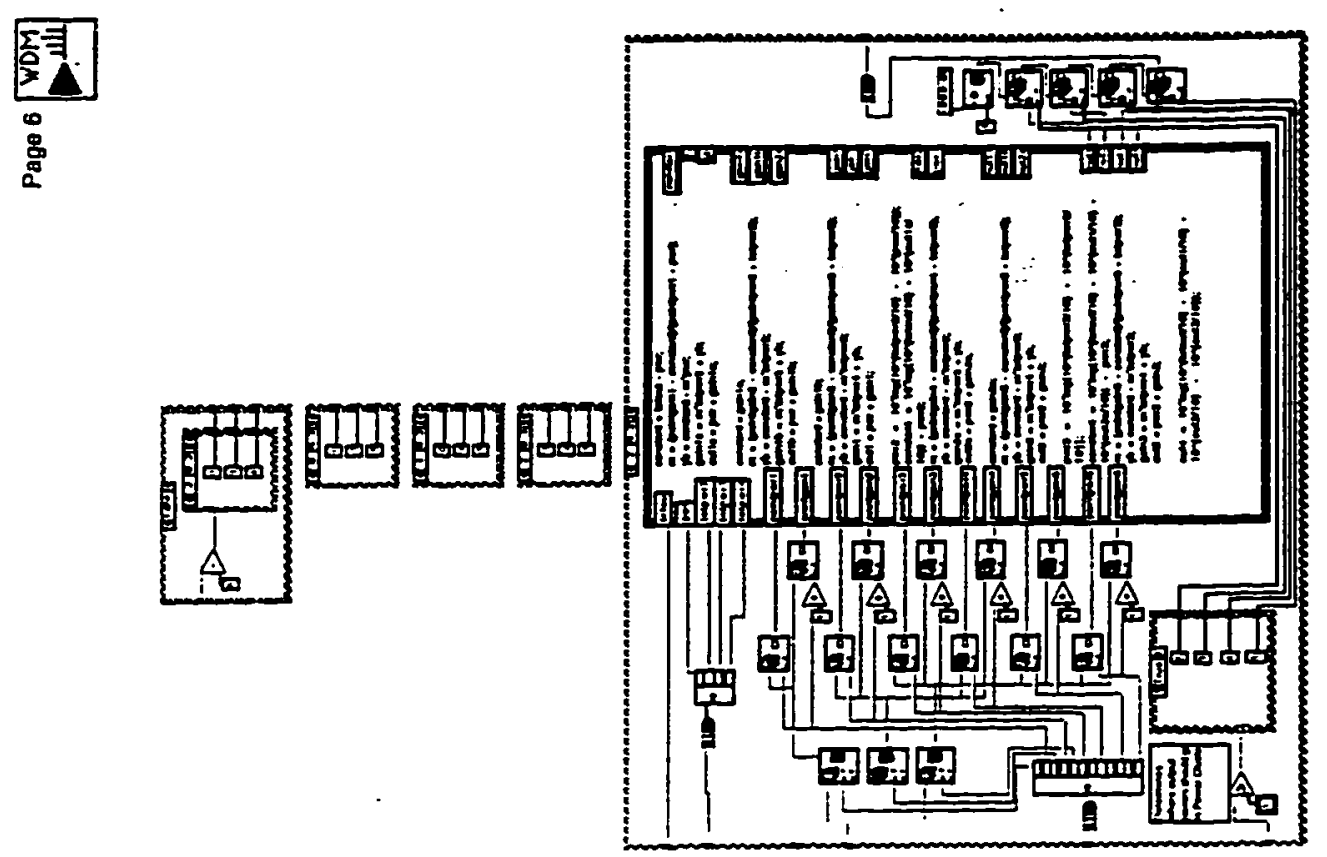

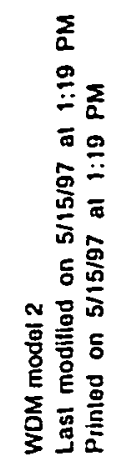

